\title{
Correlation of Transcranial Color Doppler to N20 Somatosensory Evoked Potential Detects Ischemic Penumbra in Subarachnoid Hemorrhage
}

\author{
Piero Di Pasquale*,1, Paolo Zanatta ${ }^{2}$, Ilaria Morghen ${ }^{3}$, Enrico Bosco $^{2}$ and Elena Forini ${ }^{4}$ \\ ${ }^{1}$ Anaesthesia and Intensive Care Department, Rovigo Hospital, Viale 3 Martiri, 140, 45100 Rovigo, Italy \\ ${ }^{2}$ Anaesthesia and Intensive Care Department, Treviso Regional Hospital, Viale Vittorio Veneto, 18, 31100 Treviso, Italy \\ ${ }^{3}$ Anaesthesiology and Intensive Care Department, S. Anna University Hospital, C.so Giovecca 203, 44100 Ferrara, Italy \\ ${ }^{4}$ Health Statistics Service, S. Anna University Hospital, C.so Giovecca 203, 44100 Ferrara, Italy
}

\begin{abstract}
Background: Normal subjects present interhemispheric symmetry of middle cerebral artery (MCA) mean flow velocity and N20 cortical somatosensory evoked potential (SSEP). Subarachnoid haemorrhage (SAH) can modify this pattern, since high regional brain vascular resistances increase blood flow velocity, and impaired regional brain perfusion reduces N20 amplitude. The aim of the study is to investigate the variability of MCA resistances and N20 amplitude between hemispheres in SAH.

Methods: Measurements of MCA blood flow velocity (vMCA) by transcranial color-Doppler and median nerve SSEP were bilaterally performed in sixteen patients. MCA vascular changes on the compromised hemisphere were calculated as a ratio of the reciprocal of mean flow velocity (1/vMCA) to contralateral value and correlated to the simultaneous variations of interhemispheric ratio of N20 amplitude, within each subject. Data were analysed with respect to neuroimaging of MCA supplied areas.

Results: Both interhemispheric ratios of 1/vMCA and N20 amplitude were detected $>0.65(\mathrm{p}<0,01)$ in patients without neuroimages of injury. Both ratios became $<0.65(\mathrm{p}<0.01)$ when patients showed unilateral images of ischemic penumbra and returned $>0.65$ if penumbra disappeared. The two ratios no longer correlated after structural lesion developed, as N20 detected in the damaged side remained pathological (ratio $<0.65$ ), whereas $1 /$ vMCA reverted to symmetric interhemispheric state (ratio >0.65), suggesting a luxury perfusion.

Conclusion: Variations of interhemispheric ratios of MCA resistance and cortical N20 amplitude correlate closely in SAH and allow identification of the reversible ischemic penumbra threshold, when both ratios become $<0.65$. The correlation is lost when structural damage develops.
\end{abstract}

Keywords: Subarachnoid haemorrhage, ischemic penumbra, cerebral blood flow, brain perfusion, brain vascular resistances, transcranial color-doppler, somatosensory evoked potentials.

\section{BACKGROUND}

Both Middle Cerebral Artery (MCA) mean flow velocity measured by transcranial color Doppler (TCCD) and somatosensory evoked potential (SSEP) show high interhemispheric symmetry in normal subjects [1-4]. This condition could be modified after subarachnoid haemorrhage (SAH), due to prevalent unilateral alterations in vasoregulation of cerebral arteries and vasospasm [5,6], resulting in impaired regional brain perfusion [7,8]. The vessel section $\pi r^{2}(r=$ radius of the vessel) is inversely proportional to mean blood flow velocity $\mathrm{V}$, and directly proportional to blood flow $\mathrm{F}$ $\left(\pi r^{2}=\mathrm{F} / \mathrm{V}\right)$. The variability in MCA vessel section resistances and blood flow, between hemispheres within one subject, can be evaluated by a semi quantitative approach, comparing the reciprocal of MCA mean blood flow velocities (1/vMCA) detected simultaneously on both sides, assuming the other determinants of $\mathrm{F}$ and $\mathrm{V}$ to be constant $(\mathrm{F}=$ $\Delta \mathrm{P} \cdot \pi \mathrm{r}^{2} \cdot \mathrm{r}^{2} / 8 \eta \mathrm{l}, \mathrm{V}=\Delta \mathrm{P} \cdot \pi \mathrm{r}^{2} / 8 \eta \mathrm{l}, \Delta \mathrm{P}=$ cerebral perfusion pressure, $\eta=$ blood viscosity and $1=$ length of measured

*Address correspondence to this author at the Anaesthesia and Intensive Care Department, Rovigo Hospital, Viale 3 Martiri, 140, 45100 Rovigo, Italy; Tel: +39 0425394224 +39 3356523854; Fax: 0425 394223;

E-mail: piero.dipasquale@teletu.it segments of MCA). In addiction, there is strong correlation between variations of electrophysiological changes and regional cerebral blood flow (rCBF), and a $50 \%$ reduction in cortical SSEP amplitude at rCBF levels between 14 and 16 $\mathrm{ml} / 100 \mathrm{~g} / \mathrm{min}$ has been demonstrated [9]; for this reason cortical N20 SSEP from the median nerve has been widely used to evaluate cerebral function in carotid surgery and intracranial aneurysm operative procedures [10-12]. Mechanism, diagnosis and prevention of brain injury after SAH have been widely investigated [13-20], but evaluation of correlation between variations of cortical activity expressed by evoked potentials and regional brain vascular changes detected by transcranial color-Doppler is lacking. The aim of the study is to investigate the correlation between changes in resistances due to vessel section reduction of MCA and modifications of cortical SSEP by interhemispheric comparison of both values of $1 / \mathrm{vMCA}$ detected by TCCD and N20 amplitude, in a series of case studies of patients with SAH.

\section{METHODS}

\section{Participants}

We studied retrospectively sixteen patients $(8$ males and 8 females) consecutively admitted to Rovigo Hospital Inten- 
Table 1. Description of Patients

\begin{tabular}{|c|c|c|c|c|c|c|}
\hline Patient & $\operatorname{sex}$ & $\begin{array}{c}\text { Age } \\
(\text { mean } \pm \text { S.D })\end{array}$ & $\begin{array}{l}\text { GCS on admission } \\
\quad(\text { mean } \pm \text { S.D. })\end{array}$ & Aneurism site & Fisher score & Surgery \\
\hline 1 & $\mathrm{~m}$ & 63 & 9 & Lt ACA & 4 & Clip \\
\hline 2 & $\mathrm{f}$ & 57 & 7 & Rt PComA & 3 & Clip Craniectomy EVD \\
\hline 3 & $\mathrm{f}$ & 68 & 8 & AComA & 4 & Clip Craniectomy EVD \\
\hline 4 & $\mathrm{f}$ & 69 & 4 & Rt MCA & 4 & EVD \\
\hline 5 & $\mathrm{~m}$ & 32 & 8 & Rt MCA & 3 & Clip \\
\hline 6 & $\mathrm{f}$ & 30 & 13 & No evidence & 2 & No surgery \\
\hline 7 & $\mathrm{f}$ & 69 & 7 & Rt MCA & 3 & Clip \\
\hline 8 & $\mathrm{~m}$ & 41 & 5 & A ComA & 4 & Coil Clip Craniectomy EVD \\
\hline 9 & $\mathrm{~m}$ & 48 & 8 & A ComA & 3 & Clip Craniectomy \\
\hline 10 & $\mathrm{~m}$ & 52 & 9 & Lt MCA & 3 & Clip \\
\hline 11 & $\mathrm{~m}$ & 75 & 9 & Lt MCA & 4 & Clip Craniectomy \\
\hline 12 & $\mathrm{~m}$ & 45 & 6 & No evidence & 3 & EVD \\
\hline 13 & $\mathrm{~m}$ & 46 & 12 & ACA & 2 & No surgery \\
\hline 14 & $\mathrm{f}$ & 49 & 12 & Rt MCA & 2 & Clip \\
\hline 15 & $\mathrm{f}$ & 54 & 9 & Lt ICA & 2 & Clip Craniectomy EVD \\
\hline \multirow[t]{2}{*}{16} & $\mathrm{~m}$ & 73 & 10 & Lt ACA & 2 & Clip \\
\hline & & $(54.4 \pm 14)$ & $(8.5 \pm 2.4)$ & & & \\
\hline
\end{tabular}

Lt ACA = Left Anterior Cerebral Artery, Rt PcomA = Right Posterior Communicating Artery, A comA = Anterior Communicating Artery, Rt MCA = Right Middle Cerebral Artery, Lt MCA = Left Middle Cerebral Artery, Lt ICA = Left internal Carotid Artery, EVD = External Ventricular Drainage.

sive Care and Neurosurgery Department. Inclusion criteria and eligibility: $\geq 18$ years old, diagnosis of spontaneous SAH by Digital Subtraction Angiography (DSA), absence of previous structural ischemic/haemorrhagic brain injury and stenosis of the neck and brain arteries. The average age was 54.4( $\mathrm{SD}=14)$. Reported data gathered were: Glasgow Coma Score (GCS) on admission, aneurism site, Fisher score and surgery (Table 1).

\section{Procedures and Examination Timing}

All patients were submitted to neuroimaging, SSEP and TCCD investigations along their Intensive Care Unit stay. SAH diagnosis was made by DSA and/or Computed Tomographic angiography (CTA). Subsequently, patients were evaluated with Computed Axial Tomography (CT), CTA, Magnetic Resonance Imaging (MRI) or Magnetic Resonance angiography (MRA) twelve hours after surgery and when needed on a clinical evaluation basis. Timing of first examination after SAH diagnosis of each patient (neuroimaging and simultaneous SSEP and TCCD) is showed in Table 2 and subsequent examinations in Table $\mathbf{3}$ (in the column 'day of examin. after SAH diagnosis'). SSEP by median nerve and TCCD of MCA were performed bilaterally on each patient. Preferably bilateral SSEP and TCCD should be performed simultaneously during each examination. As this was not possible, we conducted sequential recording of SSEP and TCCD in a very close temporal succession, of no longer than thirty minutes. They were considered as simultaneously recorded if the clinical conditions, monitored respiratory and hemodynamic parameters and infusion therapy were unchanged during examination. Each patient was examined several times on different days throughout the survey. Thir- teen patients were examined after surgery; one patient before and after; two did not undergo surgery during our observation. Four patients were awake during examinations. Twelve were under mechanical ventilation and sedated with a remifentanil and propofol infusion. A normal range of arterial oxygenation (oxygen arterial partial pressure $>100$ $\mathrm{mmHg}$ ), normocapnia (carbon dioxide arterial partial pressure between 35 and $40 \mathrm{mmHg}$ ), mean arterial pressure above $70 \mathrm{mmHg}$ and haemoglobin value higher than $10 \mathrm{~g} / \mathrm{dl}$ were maintained; intracranial pressure (ICP) and CPP were monitored in 7 patients only. We briefly suspended hypnotics infusion to perform the clinical evaluation of the patients after each examination.

\section{SSEP}

A Nicolet Viking Four Electrodiagnostic System, with four channel amplifier and isolated electrical stimulator, was used to obtain the evoked potentials. Surface stimulating electrodes and steel needle subcutaneous electrodes were used for recording. Recording electrodes were positioned on Erb's point, cervical spine (C7) with reference to antecollis, cortical $\mathrm{C}^{\prime}$ ' and $\mathrm{C} 4$ ' ( $2 \mathrm{~cm}$ posterior to $\mathrm{C} 3$ and $\mathrm{C} 4$, international 10-20 system) and ipsilateral mastoid with reference to Fz. SSEP stimulation parameters were $10-20 \mathrm{~mA}$ intensity (4-6 mA above visible thumb twitching threshold), $0.2 \mathrm{~ms}$ duration and $4.1 \mathrm{~Hz}$ square wave. Sweep time was at $100 \mathrm{~ms}$, sensitivity at $2 \mu \mathrm{V} / \mathrm{div}$; filters were set at 30 and $3000 \mathrm{~Hz}$. For each recording condition, two runs of 300 and 250 responses were averaged, and checked for reproducibility, without artefacts, and superimposed. Latency and amplitude of N9, N13, P14 and N20 were recorded and measured during the first examination; subsequently only N9 and N20 waves were recorded. Peaks were 
Table 2. Examination Timing

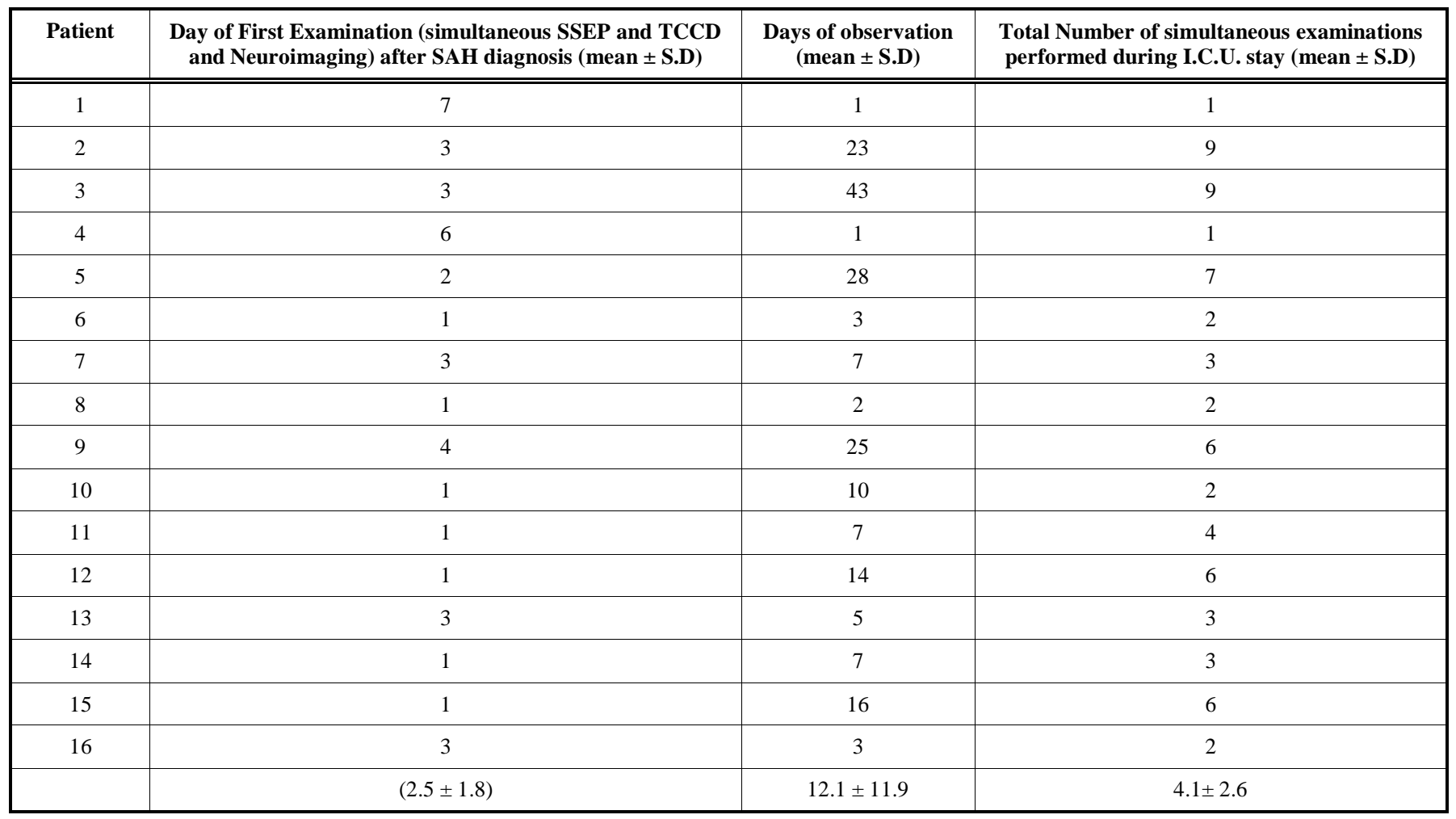

Table 3. Variations of N20 Amplitude and Reciprocal of MCA Mean Flow Velocity (1/vMCA) Detected on the Compromised Hemisphere Expressed as a Ratio of Contralateral Values, Compared to CT-MRI Scan Image of Corresponding MCA Brain Area, N20 Amplitude Absolute Value, Vasospasm and Lateralizing Symptoms

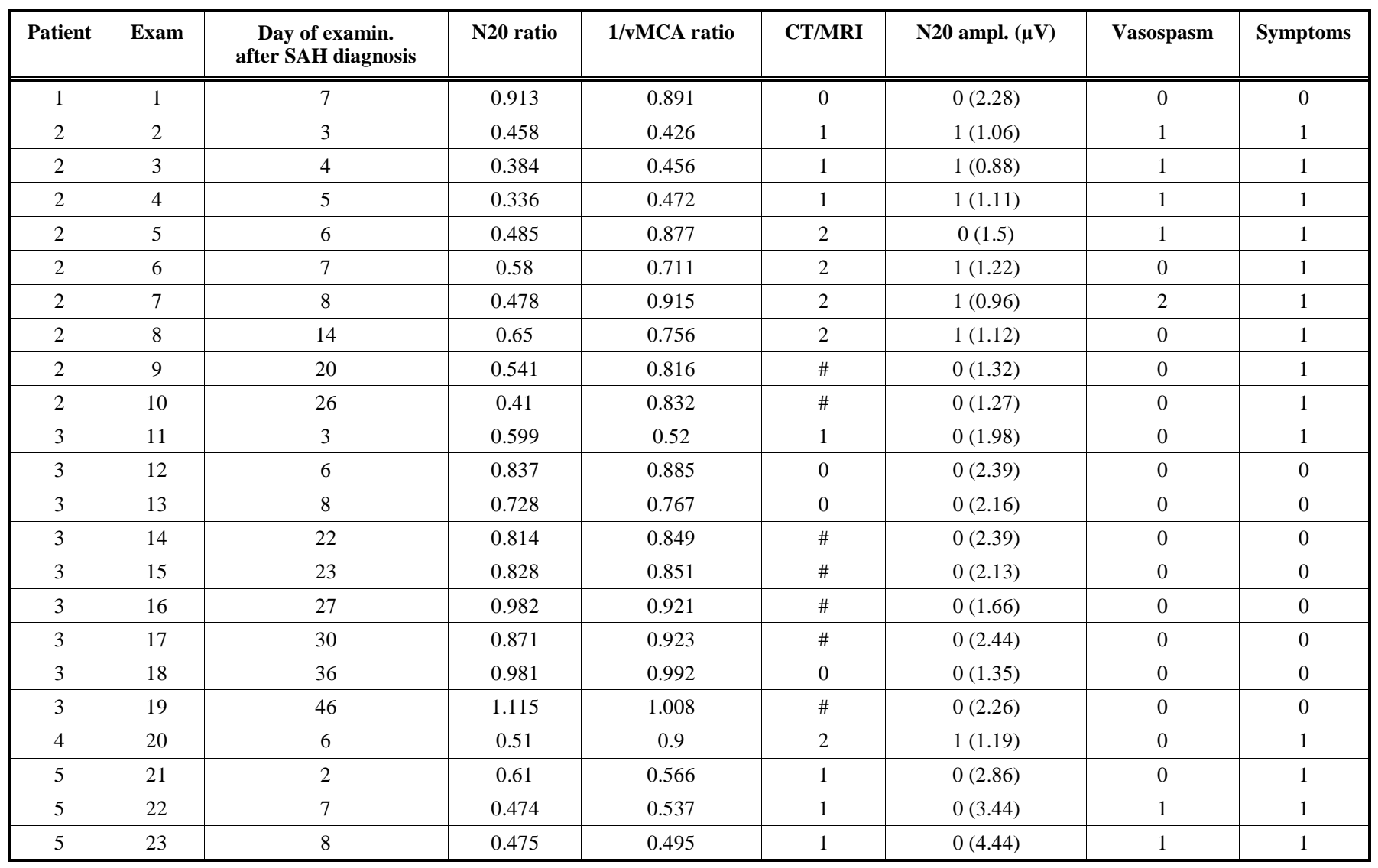


(Table 3). Contd.....

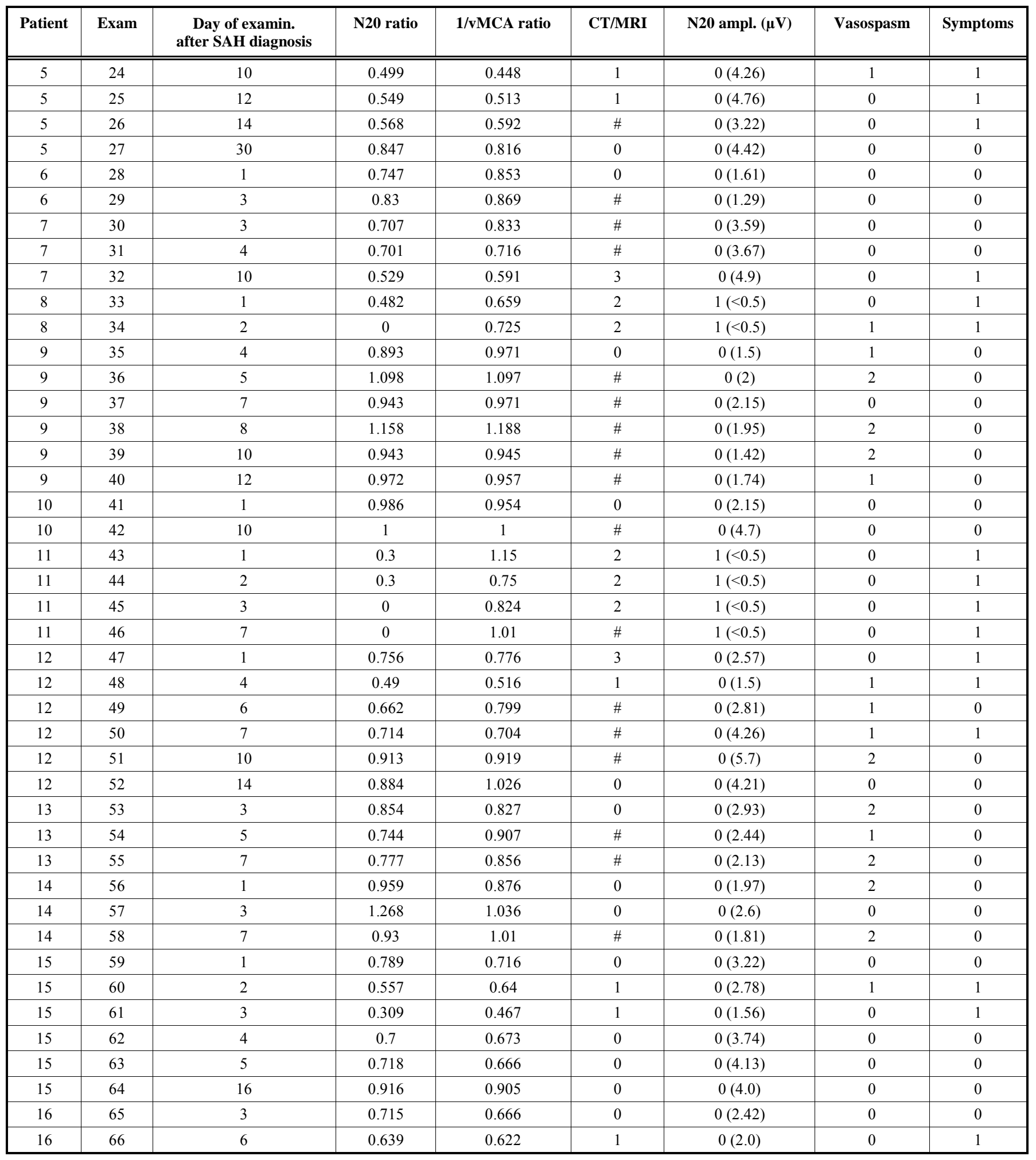

CT/MRI: MCA area alterations image: $\mathbf{0}=$ no alterations, $\mathbf{1}=$ transient and reversible lesions (oedema, hypodensity), $\mathbf{2}=$ structural damage (ischemia, hematoma), $\mathbf{3}=$ hydrocephalus, \# = missing. $\mathbf{N 2 0}$ amp : N20 amplitude on the compromised hemisphere : $\mathbf{0}=\mathrm{N} 20$ amplitude $>1,2 \mu \mathrm{V}, \mathbf{1}=\mathrm{N} 20$ amplitude $<1,2 \mu \mathrm{V}$. Vasospasm : $\mathbf{0}=$ absence, $\mathbf{1}=$ monolateral, $\mathbf{2}=$ bilateral. Symptoms : $\mathbf{0}=$ absence, $\mathbf{1}=$ presence $(\mathrm{arm}$ paresis, hemiparesis, hemiplegia or/and consciousness alteration).

indicated with cursors on the screen, placed manually; peaklatencies and peak-to-peak amplitudes were calculated on the first trace. Normal value of $\mathrm{N} 20$ amplitude was fixed at 1.2 $\mu \mathrm{V}$, as indicated by Amantini [21], using the same reference (Fz).

\section{TCCD}

Vivid 3 Expert General Electric Ultrasound Unit, equipped with $1,8-2,5 \mathrm{MHz} 90^{\circ}$ phased-array probe for both B-mode and colour Doppler imaging was used to perform TCCD examinations. Intracranial cerebral arteries were ex- 
amined bilaterally through the acoustic window in the temporal squama, and, if present, through the craniectomy window. The M1 segment of MCA was identified with colour imaging; a 2 to $6 \mathrm{~mm}$ wide sample volume was placed on the colour image of the artery at the site of the highest flow acceleration. The angle of insonation was corrected by visual guidance, orientating a linear marker provided with the scanner along the long axis of the segment. The same wide sample volume was set and used during each bilateral examination. Mean flow velocity was calculated with automatic tracing and manual tracing in case of a wake signal. Bilateral mean flow velocity of the internal carotid artery at the angle of the inferior mandible was recorded, to determine the Lindegaard Index. Vasospasm was assumed when mean flow velocity reached $120 \mathrm{~cm} / \mathrm{sec}$ in M1 segment of MCA [22] and Lindegaard Index $>3$ [23].

\section{Data Evaluation}

All examinations and measurements were performed by the same anaesthesiologist, with more than ten years of experience in critical care, five years in evoked potentials in Intensive Care Unit and two years in transcranial colorDoppler. Comparison between hemispheres, within each patient, of both 1/v MCA and N20 amplitude was made in each examination and values recorded on the compromised hemisphere were expressed as a ratio of the contralateral values. Correlation of $1 / \mathrm{vMCA}$ to $\mathrm{N} 20$ interhemispheric ratio was calculated. In addition, N20 absolute value and presence/absence of vasospasm and neurological symptoms such as drowsiness, consciousness modifications and hemiparesis/hemiplegia were evaluated; other identifiable causes of neurological worsening such as serum electrolyte or glucose disturbances, hypoxia, hypercapnia, or seizures (clinical or electrographic) were excluded. All data were analyzed with respect to Brain CT or MRI executed on same day as TCCD and SSEP examination. We considered images of transient and reversible alterations (oedema, hypodensity) or structural damage (ischemia, infarction, haemorrhage) shown in the brain area supplied by MCA; hydrocephalus treated by external ventricular drainage (EVD) has been considered as reversible alteration.

\section{Statistical Analysis}

Mean and percentage were used for descriptive purposes. Statistical significance was defined as $\mathrm{p}<0.01$. Pearson Coefficient Correlation (r), with respective confidence interval at $95 \%(95 \% \mathrm{CI})$, was used to determine quantitative variables. SAS and SPSS (Statistical Analysis System, Software Products for Statistical Solution) were used for statistical analysis.

\section{RESULTS}

During the survey all 16 patients were eligible. A total of 66 bilateral SSEP and TCCD were performed with the criteria of simultaneousness. Values of the $1 / \mathrm{v}$ MCA and N20 amplitude recorded on the compromised hemisphere of each patient, expressed as ratios of their respective contralaterals and compared to CT/MRI scan images of the corresponding MCA supplied area, N20 amplitude absolute value, presence/absence of vasospasm and lateralizing symptoms are shown in Tab.3. The 1/vMCA and N20 interhemispheric ratio of 40 examinations were analyzed in respect of $40 \mathrm{im}-$ ages of CT/MRI scan performed on the same day as the TCCD and SSEP. Sixteen examinations in eleven patients (group 1: patients No. 1,3,5,6,9,10,12,13,14,15,16) matched CT/MRI image of no brain damage in the area supplied by the MCA: all subjects showed a high level of symmetry between interhemispheric values of 1/vMCA and N20 amplitude (Fig. 1); both interside ratios were always $>0,65$ and showed correlation $(\mathrm{p}<0,01, \mathrm{r}=078,95 \% \mathrm{CI}=0,46$ to 0,92 ). In this group, MCA unilateral vasospasm (mean flow velocity $>120<150 \mathrm{~cm} / \mathrm{sec}$, L.I. $>3)$ or almost symmetric bilateral vasospasm $(<180 \mathrm{~cm} / \mathrm{sec}$, L.I. $>3)$ was detected in $18,7 \%$ of examinations; N20 amplitudes were still bilaterally normal $(>1,2 \mu \mathrm{V})$ and symmetric. Patients did not show lateralizing neurological defects or consciousness alteration at clinical evaluation (Table 4). Fourteen examinations in seven patients (group 2: patients No. 2,3,5,7,12,15,16) matched CT/MRI images of tissue alterations in unilateral MCA area (shift, oedema, tissue hypodensity, cloth) or hydrocephalus. Both values of 1/vMCA and N20 amplitude on the compromised side were lower than contralateral and both interhemi-

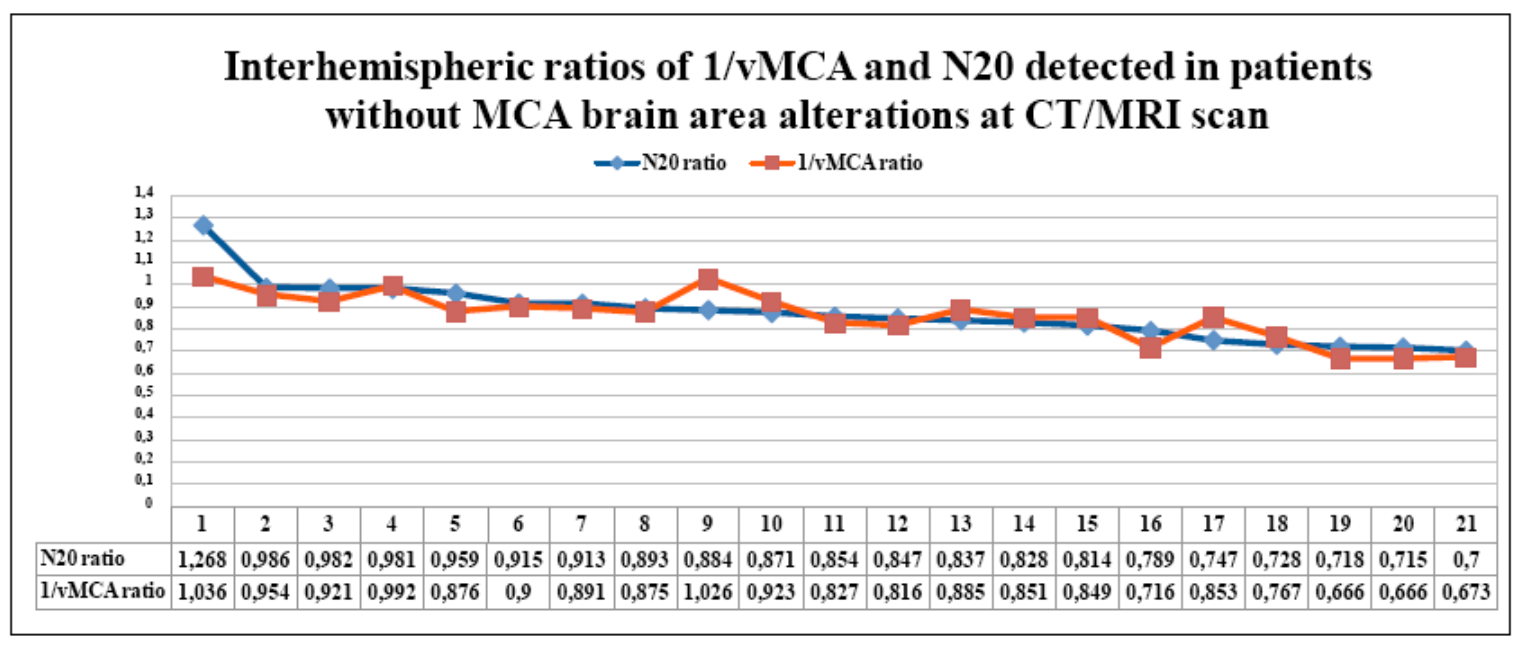

Fig. (1). The couples of $\mathrm{N} 20$ and 1/vMCA interhemispheric ratio values, detected in each patient without CT/MRI scan alterations in the MCA supplied area, gathered in Table 4, are displayed in the graph in descending order. All N20 and 1/vMCA interhemispheric ratio values were $>0.65$ and showed significant statistical correlation $(\mathrm{p}<0.01, \mathrm{r}=0.78,95 \%$ C.I. $=0.46$ to 0.92$)$. 
Table 4. Sixteen Examinations in Eleven Patients (Group 1: Patients No. 1,3,5, 6,9,10, 12,13,14,15,16) Matched CT/MRI Image of no Brain Damage in the Area Supplied by the MCA

\begin{tabular}{|c|c|c|c|c|c|c|c|}
\hline Patient & Exam & $\mathrm{N} 20$ ratio & 1/vMCA ratio & CT/MRI & N20_amp & Vasospasm & Symptoms \\
\hline 1 & 1 & 0.913 & 0.891 & 0 & 0 & 0 & 0 \\
\hline 3 & 13 & 0.728 & 0.767 & 0 & 0 & 0 & 0 \\
\hline 3 & 18 & 0.981 & 0.992 & 0 & 0 & 0 & 0 \\
\hline 6 & 28 & 0.747 & 0.853 & 0 & 0 & 0 & 0 \\
\hline 9 & 35 & 0.893 & 0.971 & 0 & 0 & 1 & 0 \\
\hline 10 & 41 & 0.986 & 0.954 & 0 & 0 & 0 & 0 \\
\hline 12 & 52 & 0.884 & 1.026 & 0 & 0 & 0 & 0 \\
\hline 14 & 57 & 1.268 & 1.036 & 0 & 0 & 0 & 0 \\
\hline 15 & 59 & 0.789 & 0.716 & 0 & 0 & 0 & 0 \\
\hline 15 & 63 & 0.718 & 0.666 & 0 & 0 & 0 & 0 \\
\hline 15 & 64 & 0.916 & 0.905 & 0 & 0 & 0 & 0 \\
\hline 16 & 65 & 0.715 & 0.666 & 0 & 0 & 0 & 0 \\
\hline
\end{tabular}

CT/MRI: MCA area alterations image $\mathbf{0}=$ no alterations, $\mathbf{1}=$ transient and reversible lesions (oedema, hypodensity), $\mathbf{2}=$ structural damage (ischemia, hematoma), $\mathbf{3}=$ hydrocephalus N20 amp : $\mathbf{0}=\mathrm{N} 20$ amplitude $>1,2 \mu \mathrm{V}, \mathbf{1}=\mathrm{N} 20$ amplitude $<1,2 \mu \mathrm{V}$. Vasospasm : $\mathbf{0}=$ absence, $\mathbf{1}=$ monolateral, $\mathbf{2}=$ bilateral. Symptoms : $\mathbf{0}=$ absence, $\mathbf{1}=$ presence $(\operatorname{arm}$ paresis, hemiparesis, hemiplegia or/and consciousness alterations).

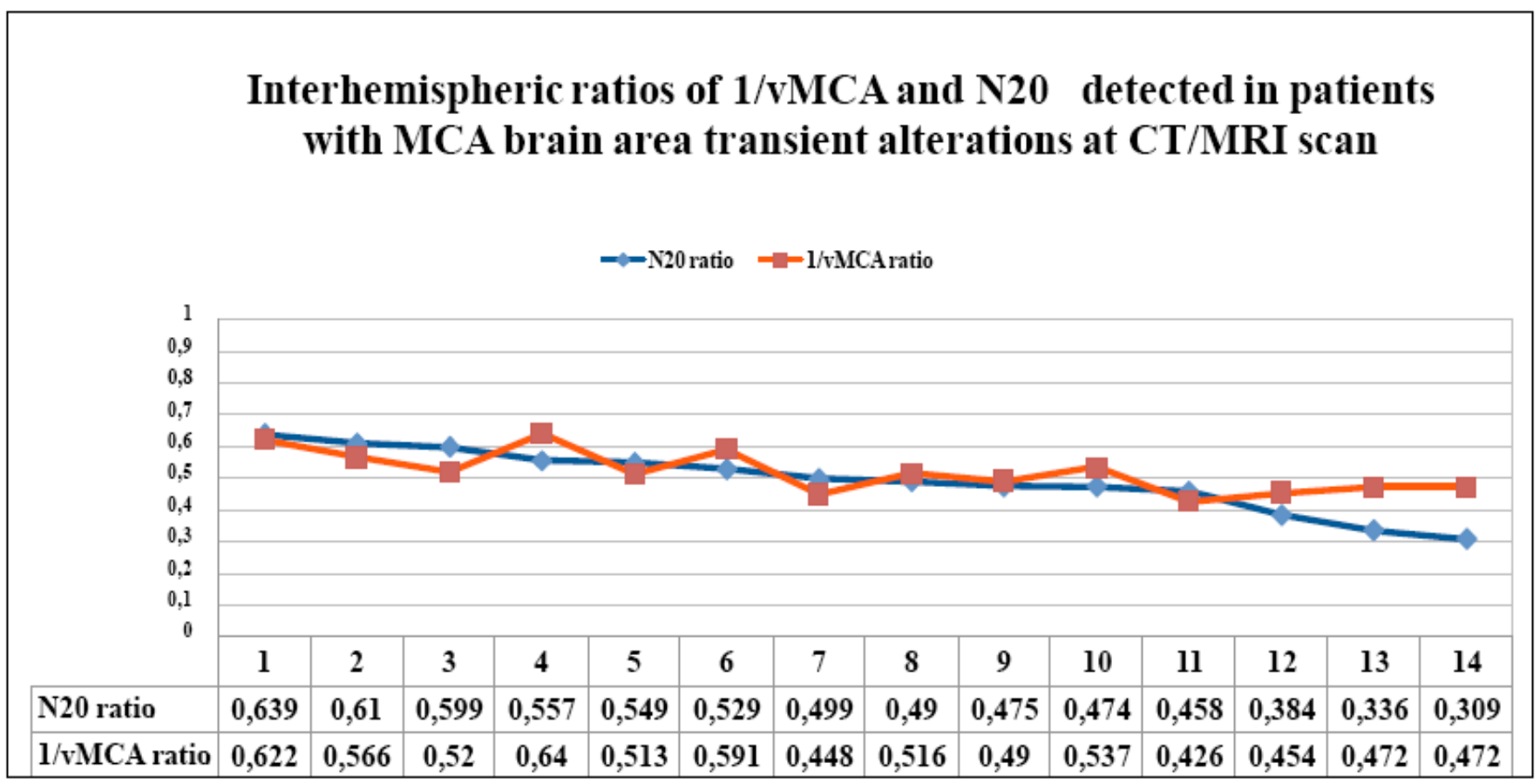

Fig. (2). The couples of $\mathrm{N} 20$ and 1/vMCA interhemispheric ratio values, detected in each patient with CT/MRI scan unilateral transient alterations in the MCA supplied area, gathered in table 5, are displayed in the graph in descending order. All N20 and 1/vMCA interhemispheric ratio values were $<0.65$ and showed significant statistic correlation $(\mathrm{p}<0.01, \mathrm{r}=0.67,95 \%$ C.I. $=0.23$ to 0.88$)$. 
Table 5. Fourteen Examinations in Seven Patients (Group 2: Patients No. 2,3,5,7, 12,15,16) Matched CT/MRI Images of Tissue Alterations in Unilateral MCA Area (Shift, Oedema, Tissue Hypodensity, Clot) or Hydrocephalus

\begin{tabular}{|c|c|c|c|c|c|c|c|}
\hline Patient & Exam & N20 ratio & 1/vMCA ratio & CT/MRI & N20_amp & Vasospasm & Symptoms \\
\hline 2 & 2 & 0.458 & 0.426 & 1 & 1 & 1 & 1 \\
\hline 2 & 3 & 0.384 & 0.456 & 1 & 1 & 1 & 1 \\
\hline 3 & 11 & 0.599 & 0.52 & 1 & 0 & 0 & 1 \\
\hline 5 & 21 & 0.61 & 0.566 & 1 & 0 & 0 & 1 \\
\hline 5 & 23 & 0.475 & 0.495 & 1 & 0 & 1 & 1 \\
\hline 5 & 24 & 0.499 & 0.448 & 1 & 0 & 1 & 1 \\
\hline 5 & 25 & 0.549 & 0.513 & 1 & 0 & 0 & 1 \\
\hline 7 & 32 & 0.529 & 0.591 & 3 & 0 & 0 & 1 \\
\hline 16 & 66 & 0.639 & 0.622 & 1 & 0 & 0 & 1 \\
\hline
\end{tabular}

CT/MRI: MCA area alterations image $\mathbf{0}=$ no alterations, $\mathbf{1}=$ transient and reversible lesions (oedema, hypodensity), $\mathbf{2}=$ structural damage (ischemia, hematoma) $\mathbf{3}=$ hydrocephalus N20 amp : $\mathbf{0}=\mathrm{N} 20$ amplitude $>1,2 \mu \mathrm{V}, \mathbf{1}=\mathrm{N} 20$ amplitude $<1,2 \mu \mathrm{V}$. Vasospasm : $\mathbf{0}=$ absence, $\mathbf{1}=$ monolateral, $\mathbf{2}=$ bilateral. Symptoms : $\mathbf{0}=$ absence, $\mathbf{1}=$ presence $(\operatorname{arm}$ paresis, hemiparesis, hemiplegia or/and consciousness alterations).

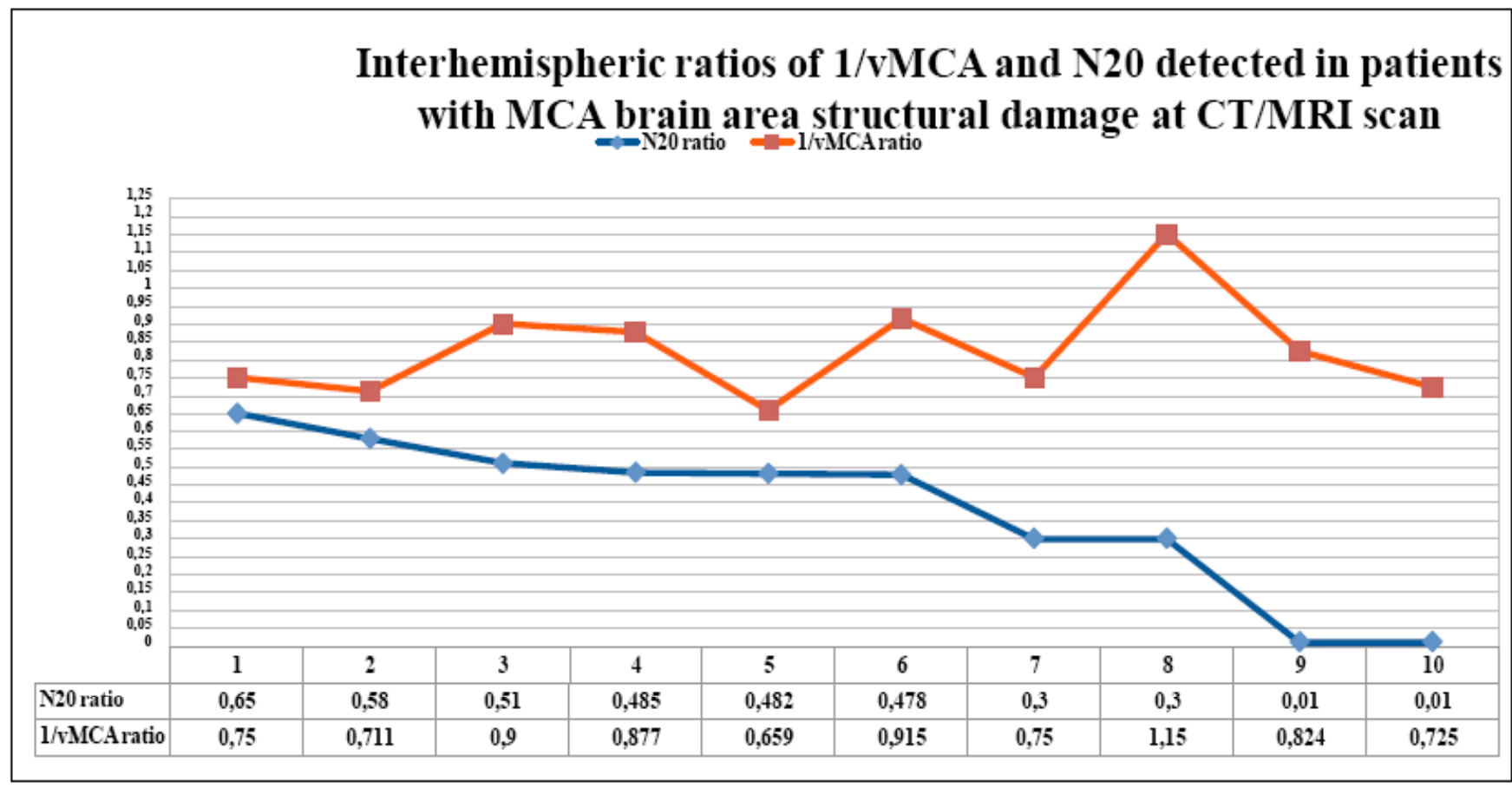

Fig. (3). The couples of N20 and 1/vMCA interhemispheric ratio values detected in each patient with CT/MRI scan unilateral structural ischemic or hemorrhagic alterations in the MCA supplied area, gathered in table 6, are displayed in the graph in descending order of N20 ratio values. The two ratios show divergent courses, since the value of $\mathrm{N} 20$ on the compromised hemisphere is $<0.65$ with respect to the contralateral, whereas the $1 / \mathrm{vMCA}$ value reverts to ratio $>0.65$. The two ratios no longer correlate in this group $(\mathrm{p}=0.88, \mathrm{r}=-0.05,95 \%$ C.I. $=-$ 0.66 to 0.59$)$.

spheric ratios were always $<0,65$ in all these patients ( $\mathrm{p}$ $<0,01 \mathrm{r}=0,67,95 \% \mathrm{CI}=0,23$ to 0,88$)$ (Fig. 2). Unilateral MCA vasospasm (mean flow velocity $>120<180 \mathrm{~cm} / \mathrm{sec}$ and L.I. $>3$ ) was detected in $57 \%$ of overall examinations.
N20 amplitudes on the compromised hemisphere were much lower than those on the contralateral in all patients, but still $>$ $1,2 \mu \mathrm{V}$, except in patient No.2, who reached pathological amplitude $(<1,2 \mu \mathrm{V})$ in several examinations. Corresponding 
Table 6. Ten Examinations in Four Patients (Group 3: No. 2,4,8,11) Matched Unilateral CT/MRI Brain Structural Ischemic or Hemorrhagic Damage Evidence

\begin{tabular}{|c|c|c|c|c|c|c|c|}
\hline Patient & Exam & $\mathrm{N20}$ ratio & 1/vMCA ratio & CT/MRI & N20_amp & Vasospasm & Symptoms \\
\hline 2 & 5 & 0.485 & 0.877 & 2 & 0 & 1 & 1 \\
\hline 2 & 6 & 0.58 & 0.711 & 2 & 1 & 0 & 1 \\
\hline 2 & 7 & 0.478 & 0.915 & 2 & 1 & 2 & 1 \\
\hline 2 & 8 & 0.65 & 0.756 & 2 & 1 & 0 & 1 \\
\hline 4 & 20 & 0.51 & 0.9 & 2 & 1 & 0 & 1 \\
\hline 8 & 33 & 0.482 & 0.659 & 2 & 1 & 0 & 1 \\
\hline 8 & 34 & 0 & 0.725 & 2 & 1 & 1 & 1 \\
\hline 11 & 43 & 0.3 & 1.15 & 2 & 1 & 0 & 1 \\
\hline 11 & 44 & 0.3 & 0.75 & 2 & 1 & 0 & 1 \\
\hline 11 & 45 & 0 & 0.824 & 2 & 1 & 0 & 1 \\
\hline
\end{tabular}

CT/MRI: MCA area alterations image $\mathbf{0}=$ no alterations, $\mathbf{1}=$ transient and reversible lesions (oedema, hypodensity), $\mathbf{2}=$ structural damage (ischemia, hematoma), $\mathbf{3}=$ hydrocephalus. N20 amp : $\mathbf{0}=\mathrm{N} 20$ amplitude $>1,2 \mu \mathrm{V}, \mathbf{1}=\mathrm{N} 20$ amplitude $<1,2 \mu \mathrm{V}$. Vasospasm : $\mathbf{0}=$ absence, $\mathbf{1}=$ monolateral, $\mathbf{2}=$ bilateral. Symptoms : $\mathbf{0}=$ absence, $\mathbf{1}=$ presence $($ arm paresis, hemiparesis, hemiplegia or/and consciousness alterations).

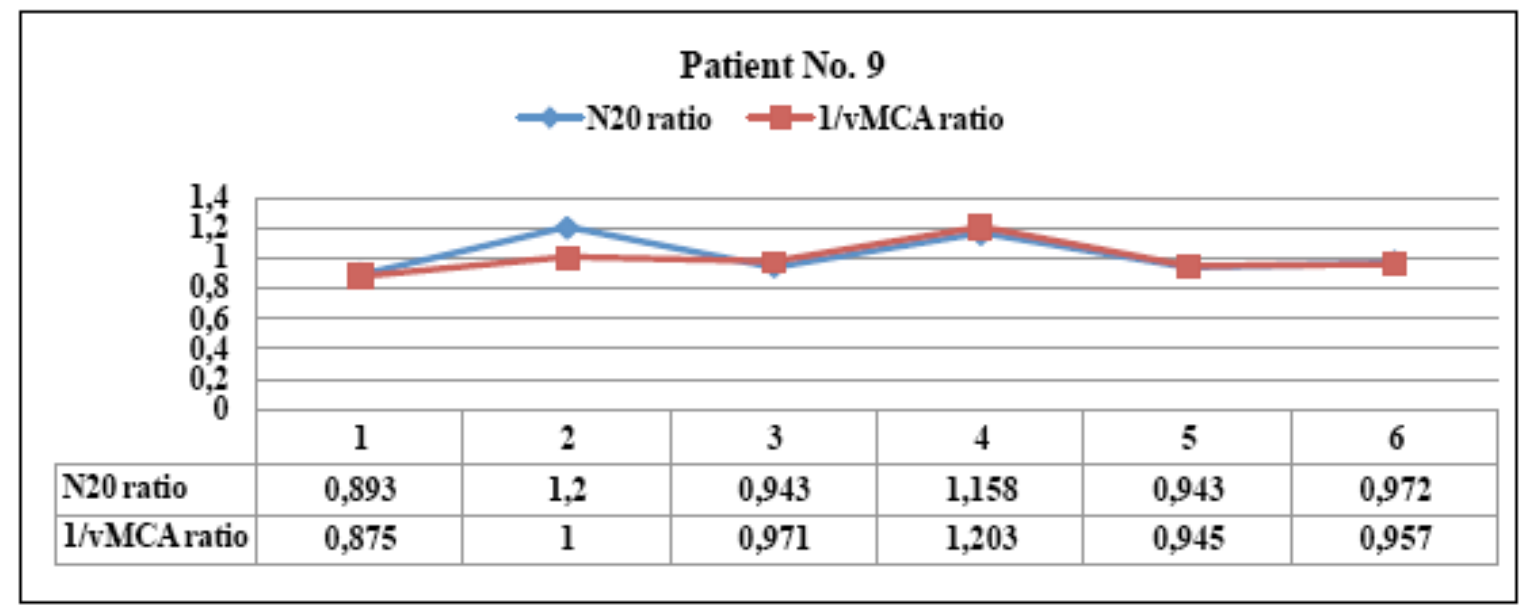

Fig. (4). Patient No. 9 showed parallel courses of N20 and 1/vMCA interhemispheric ratios ( always $>0.65$ ), with significant statistical correlation ( $\mathrm{p}<0.01, \mathrm{r}=0.94$, C.I. 0.57 to 0.99 ). This patient never developed CT/MRI scan alteration of MCA area. He showed transient vasospasm, even bilateral, (BFV $>120<240)$, normal N20 amplitude and was always asymptomatic during the survey.

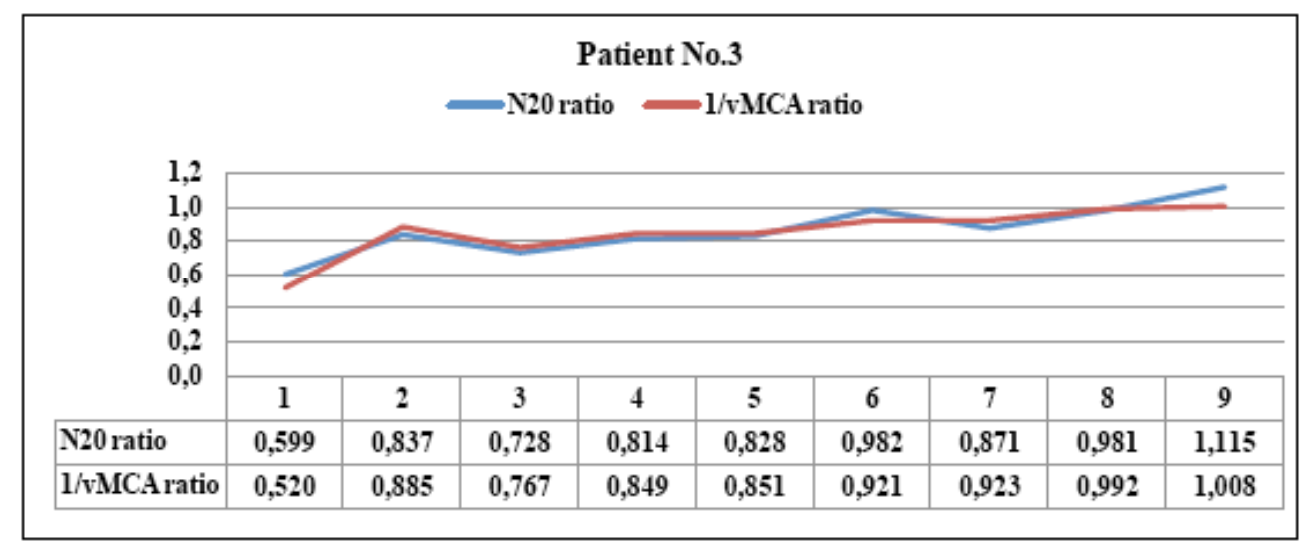

Fig. (5). Patient No. 3 showed parallel courses of N20 and 1/vMCA interhemispheric ratios with significant statistical correlation $(p<0.01$, $r$ $=0.91$, C.I. 0.64 to 0.98 ). The patient showed TC scan image of parietal oedema corresponding to both ratio values $<0.65$ in the first examination (point 1); this promptly recovered to ratio values $>0.65$ after oedema disappeared (point 2 ). This patient never reached the vasospasm threshold, always maintained N20 amplitude $>1,2 \mu \mathrm{V}$ on the compromised hemisphere and showed clinical evidence of lateralizing strength deficit with respect to the first examination only (point 1). 


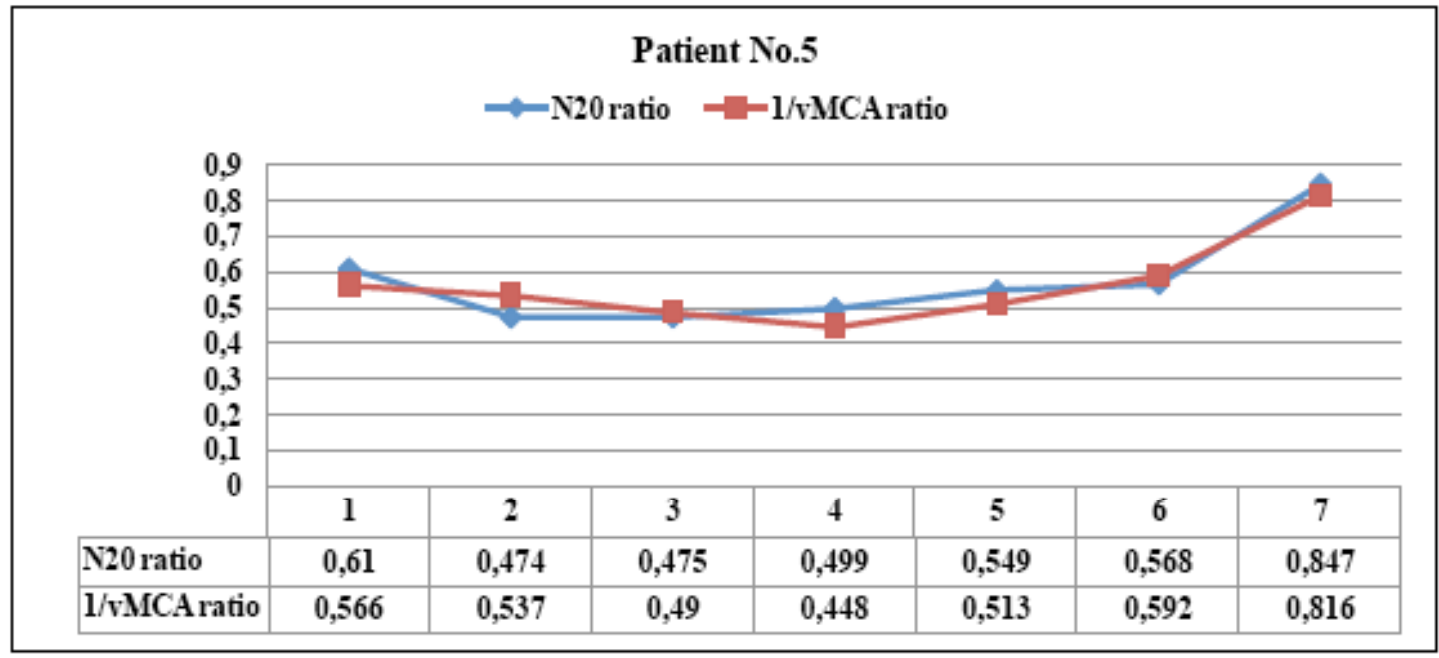

$\mathbf{a}$

Patient No. 5

Point 3

(examn.23 intab.3)

\section{Rt $/$ Lt N20 ratio $=0.475$}
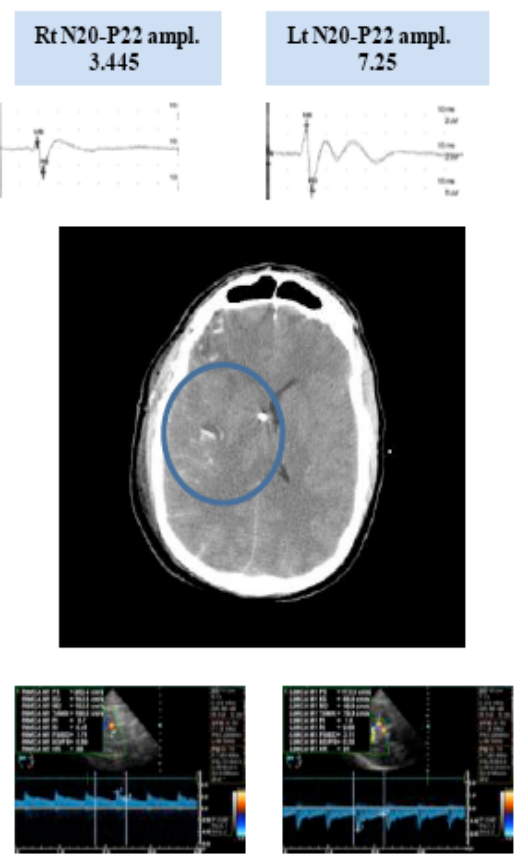

Rt l/vMCA : $1 / 160$

Rt $/$ Lt $1 /$ vMCA ratio $=0.495$
Point 4

(exam.n.24 intab.3)

Rt/Lt N20 ratio $=0.499$
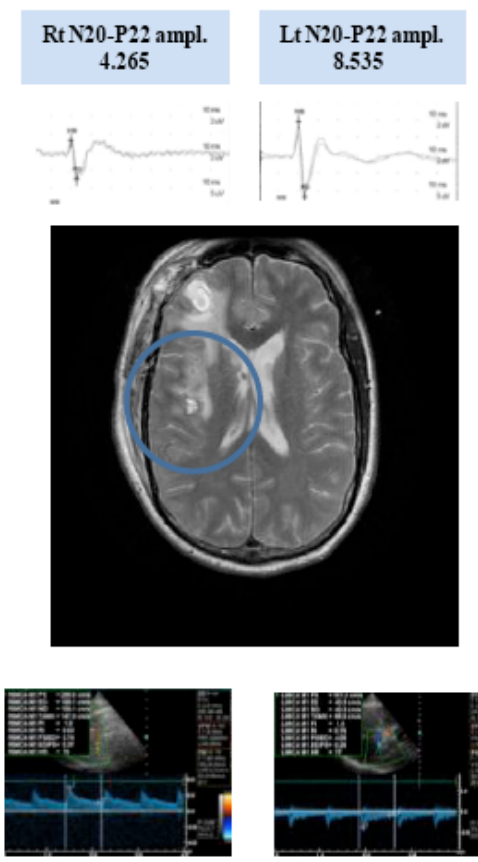

Rt 1/vMCA : $1 / 147$

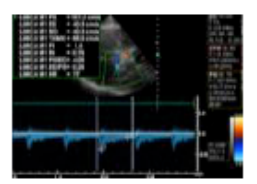

Lt 1/vMCA : $1 / 66$

Rt $/$ Lt $1 /$ vMCA ratio $=0,448$
Point 7

(exam.n.27 intab.3)

\section{Rt/Lt N20 ratio $=0.847$}
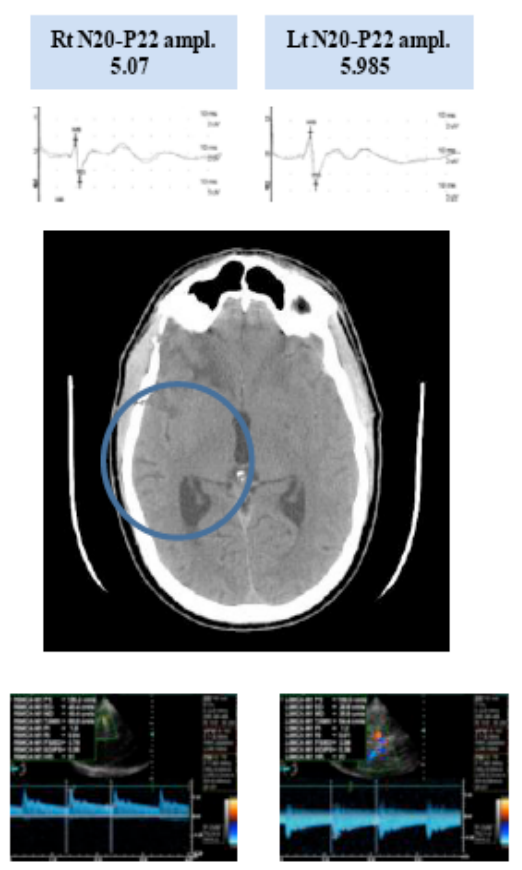

Rt 1/vMCA : $1 / 66.6$

Lt 1/vMCA : $1 / 54.4$

$\mathrm{Rt} / \mathrm{Lt} 1 / \mathrm{vMCA}$ ratio $=0.816$

b

Fig. (6). Patient No. 5: the courses of N20 and 1/vMCA ratios correlate $(\mathrm{p}<0.01, \mathrm{r}=0.94$, C.I. 0.65 to 0.99$)$. He showed both ratios $<0.65$ correspondig to points 1 to 6 (a) and and CT/MRI images of parietal oedema or hypodensity (b, points 3 and 4 ); the last couple of values $>$ 0.65 (a, point 7) correspond with a re-established normal CT scan (b, point 7). He had vasospasm $(\mathrm{BFV}>120<180)$ corresponding to points 2,3 and 4; he always maintained N20 absolute value $>1,2 \mu \mathrm{V}$ on the compromised hemisphere; when both ratios reached a value $<0.65$ (points 2 to 6), he showed lateralizing symptoms and/or consciousness modification; when both ratios reverted to a value $>0.65$ (point 7 ) he recovered to a normal clinical condition. (N20 amplitude is expressed in $\mu \mathrm{V}$ and MCA blood flow velocity in $\mathrm{cm} / \mathrm{sec}$ ). 


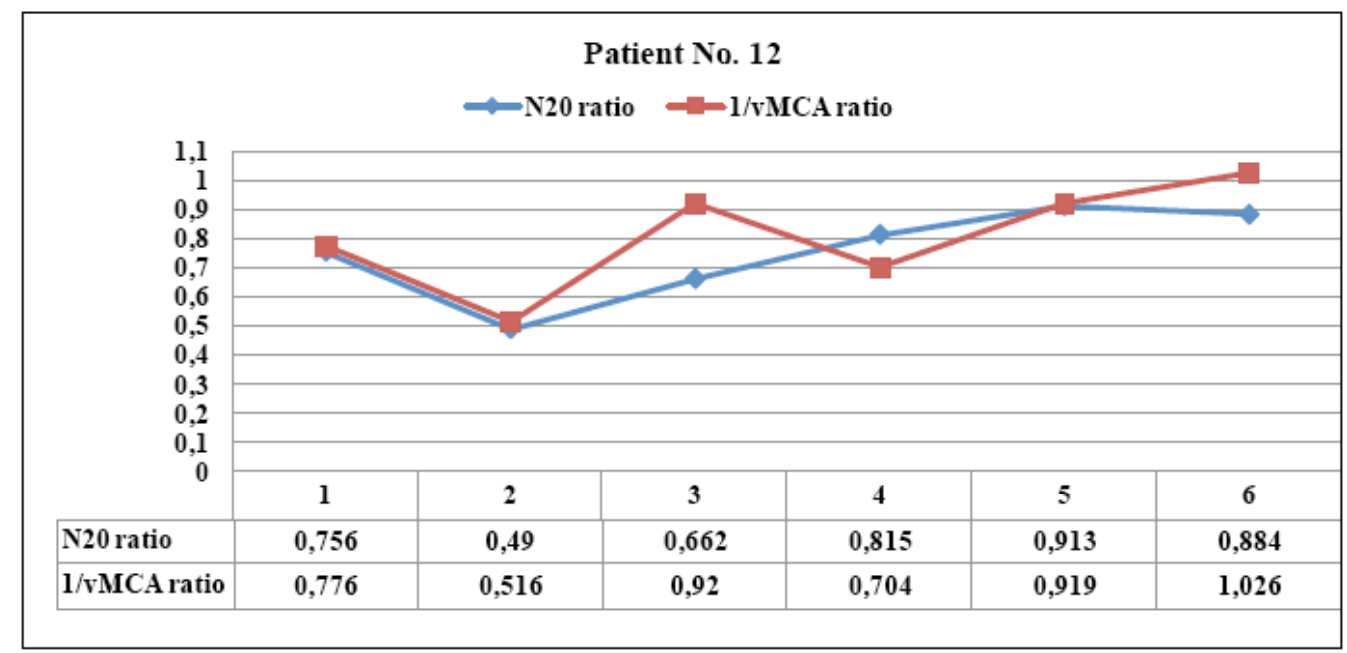

Fig. (7). Patient No. 12 showed parallel courses of $\mathrm{N} 20$ and 1/vMCA ratios ( $<<0.01, r=0.92$, C.I. $=0.44$ to 0.99 ). He showed hydrocephalus promptly treated with EVD (point 1) and right parietal oedema (point 2) in MCA area at CT scan (both ratios $<0.65$ ). He maintained N20 amplitude $>1.2 \mu \mathrm{V}$ on the compromised hemisphere and vasospasm (BFV $>120$ to $180 \mathrm{~cm} / \mathrm{sec}$ ) was detected corresponding to points $2,3,4,5$; the patient had clinical transient lateralizing symptoms corresponding to point 2 and recovered a normal clinical picture in subsequent examinations (points 3 to 6 ); CT scan performed at point 6 was normal (both ratios $>0.65$ ).

lateralizing neurological defect of muscular strength or conciousness alteration was detected at clinical examination in all these patients (Table 5). Ten examinations in four patients (group 3: patients No.2,4,8,11) matched unilateral CT/MRI brain structural ischemic or hemorrhagic damage evidence; in all of them, resistance (1/vMCA) on the compromised side reverted to a high ratio to the contralateral $(>0,65)$ and no longer correlated to the persistent interhemispheric asymmetry of N20 amplitude (ratio < 0,65) (Fig. 3); in this group correlation between the interhemispheric ratios of $1 / \mathrm{MCA}$ and $\mathrm{N} 20$ was lost $(\mathrm{p}=0.88, \mathrm{r}=-0.05,95 \% \mathrm{CI}=-0.66$ to 0.59 ). MCA vasospasm (mean flow velocity $>120<165 \mathrm{~cm}$ $/ \mathrm{sec}$ ) was detected in $33,3 \%$ of examinations. The $\mathrm{N} 20$ value on the compromised hemispheres was pathologic $(<1,2 \mu \mathrm{V})$ or absent in 9 examinations and all patients had stable hemiparesis or hemiplegia (Table 6). Patients No. 2, 3,5,12,15 belong to more than one group at different times, as a consequence of modification of their brain CT/MRI images during the survey; patient No.2 shifted from group 2 to 3; patients No. 3 and No.5 shifted from group 2 to 1; patients No.12 and No.15 shifted from group 1 to 2 and back to 1 again. In addition, we examined the course and correlation between the two interhemispheric ratios in those patients, seven in all, who were submitted to more than three examinations on different days. Statistic correlation $(\mathrm{p}<0.01)$ emerged in patient No. 9 (Fig. 4), who had no CT modifications and in those who showed transient unilateral alterations on CT/MRI in MCA supplied brain area and resumed a normal picture (patients No. 3, 5,12,15; Figs. 5-8). Interhemispheric modifications of 1/vMCA and N20 amplitude did not correlate in patients who had morphological brain damage on CT scan immediately after SAH (patient No.11, p = 0.89) (Fig. 9) or who subsequently developed brain injury (patient No.2, $\mathrm{p}=$ 0.2) (Fig. 10a,b).

\section{DISCUSSION}

The coupling between neuronal activity and its blood supply is of critical importance to the physiology of the human brain. Subarachnoid hemorrhage causes high rates of neurological deficits following impaired regional brain perfusion (15), mainly due to alterations of vascular resistances and pressure autoregulation. The vessel section, $\pi r^{2}$, is inversely proportional to mean blood flow velocity $\mathrm{V}$, and directly proportional to blood flow $F\left(\pi r^{2}=F / V\right)$. In simultaneous detection of bilateral MCA mean blood flow velocities, within one subject, we can assume constant the CPP, blood viscosity and length of measured segments of MCA. Consequently, changes in vascular resistance and blood flow, between hemispheres, due to vessels section reduction of MCA, can be evaluated in an indirect way, by a semi quantitative approach, comparing 1/vMCA detected simultaneously on both sides. Likewise, interhemispheric differences in cortical activity of the primary somatosensory area can be evaluated comparing the amplitude of the N20 waves. Our data demonstrate close correlation between the variations of 1/vMCA and cortical N20 amplitude, matching their respective interhemispheric ratios. Modifications of interhemispheric ratio of $1 / \mathrm{vMCA}$ correspond with analogue variations of interhemispheric ratio of N20 amplitude in a wide range of values, from 0.9 to $0.4-0.3(\mathrm{p}<0.01)$, unless structural ischemic or haemorrhagic damage develops (Fig. 11). When 1/vMCA and N20 amplitude had symmetric interhemispheric values (both ratios >0.65) CTI/MRI scan showed absence of alterations in the MCA areas. When both values of $1 / \mathrm{vMCA}$ and $\mathrm{N} 20$ amplitude on the compromised hemisphere became $<0.65$ of the contralateral, CT/MRI scan images showed transient modifications in the affected MCA supplied brain area and clinical evaluation revealed corresponding lateralizing symptoms or consciousness alteration (patients No. 2,3,5,12,15); consequently this data seems to identify the ischemic risk threshold. This condition of ischemic penumbra is potentially reversible, until variations of 1/MCA and N20 amplitude correlate, even at interhemispheric ratio values of $0.3-0.4$, if such extreme variations prevail only for a short time. In fact, when both $1 / \mathrm{vMCA}$ and N20 amplitude were observed to have regained ratio $>0.65$ to contralateral values (patients No. 3, 5, 12, 15), the CT/MRI scan images of MCA area and clinical condition returned to 


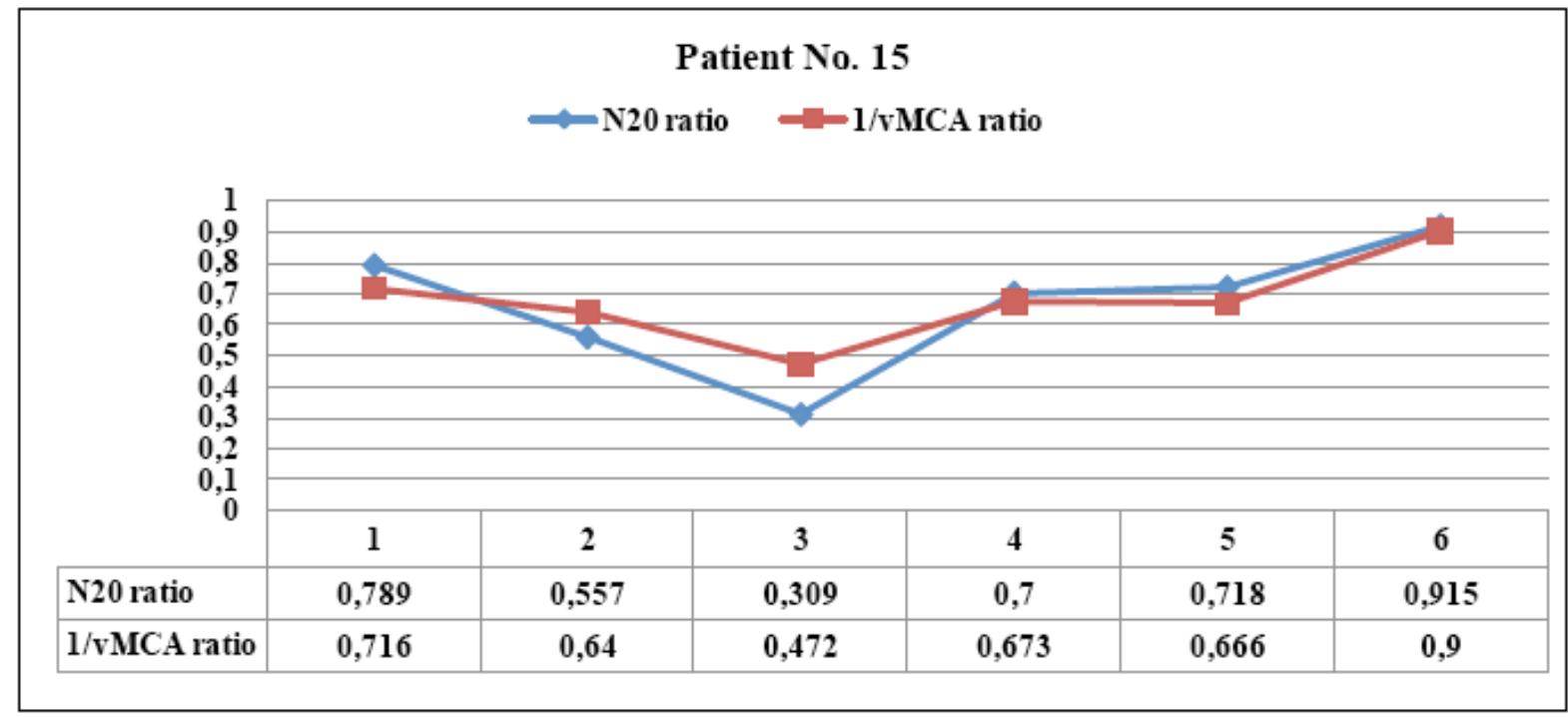

a

\section{Patient No. 15}

Point 1

(examn.59 in tab.3)

$\mathrm{Lt} / \mathrm{Rt} \mathrm{N} 20$ ratio $=0,789$
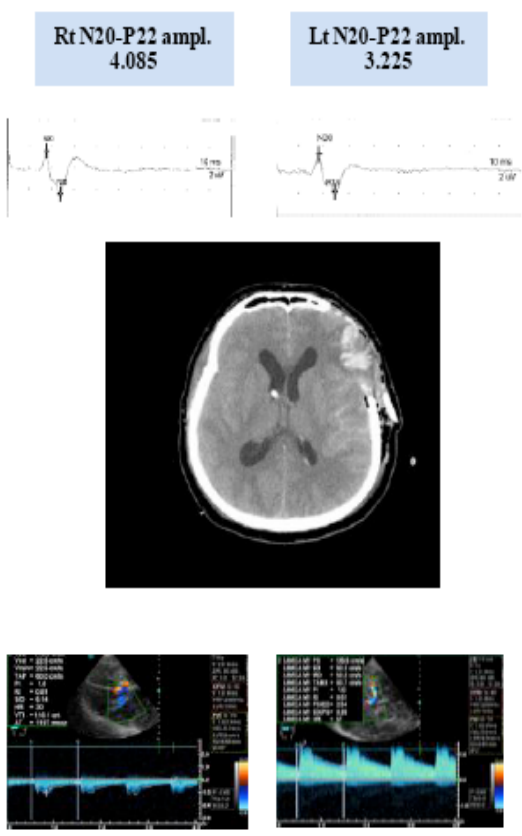

Rt l/vMCA :

$1 / 60$

Lt $/$ Rt $1 /$ vMCA ratio $=0,716$
Point 3

(exam.n6lintab. 3)

$\mathrm{Lt} / \mathrm{Rt} \mathrm{N} 20$ ratio $=0,309$
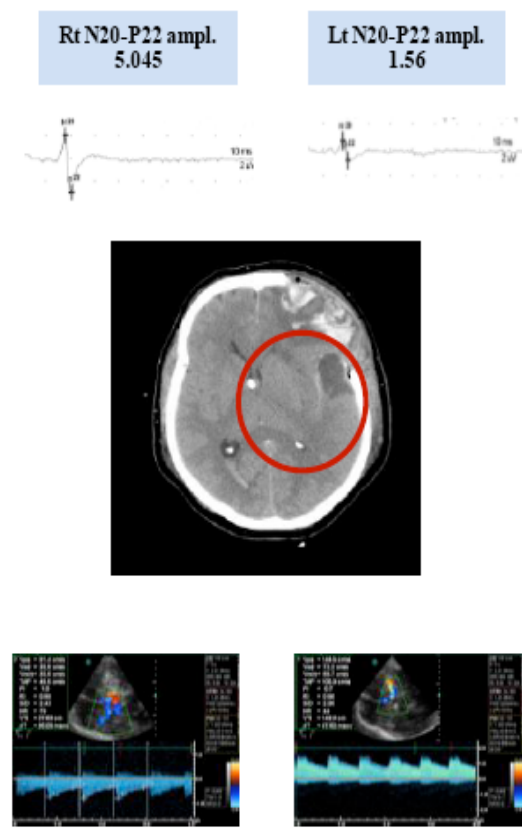

Rt 1/vMCA :

$1 / 49.5$

Lt $/$ Rt $1 /$ vMCA ratio $=0,467$
Point 6

(examn.64 in tab. 3)
$\mathrm{Lt} / \mathrm{Rt} \mathrm{N20}$ ratio $=0.916$

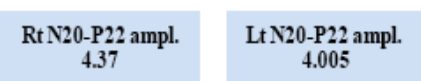

t

$+$
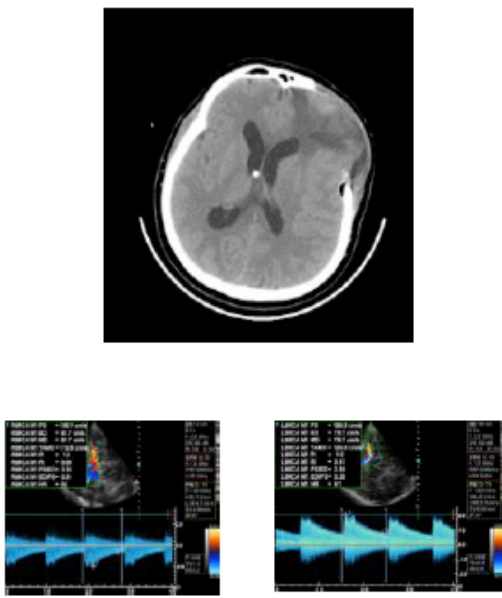

Rt 1/vMCA :

$1 / 112.6$

Lt 1/vMCA :

$1 / 124.3$

Lt $/$ Rt $1 /$ vMCA ratio $=0,905$

b

Fig. (8). Patient No. 15: the parallel variations of N20 and 1/vMCA ratio (a) show a statistically significant correlation ( $p<0.01, r=0.94$, C.I. 0.58 to 0.99 ); at first examination both ratio values $>0.65$ (a, point 1) corresponded with normal MCA supplied brain area at CT scan (b, point 1); later modifications of both ratios to values $<0.65$ (a, points 2,3 ) corresponded with the appearance of an image of parietal hypodensity at CT scan (b, point 3 ); the following ratio values $>0.65$ corresponded to normalized CT scan (b, point 6 ). This patient had vasospasm at point 2 (BFV $>180<200 \mathrm{~cm} / \mathrm{sec}$ ), had constant $>1.2 \mu \mathrm{V} \mathrm{N} 20$ amplitude, and showed lateralizing symptoms and/or consciousness modification at points 2 and 3 (ratios $<0.65$ ). (N20 amplitude is expressed in $\mu \mathrm{V}$ and MCA blood flow velocity in $\mathrm{cm} / \mathrm{sec}$ ). 


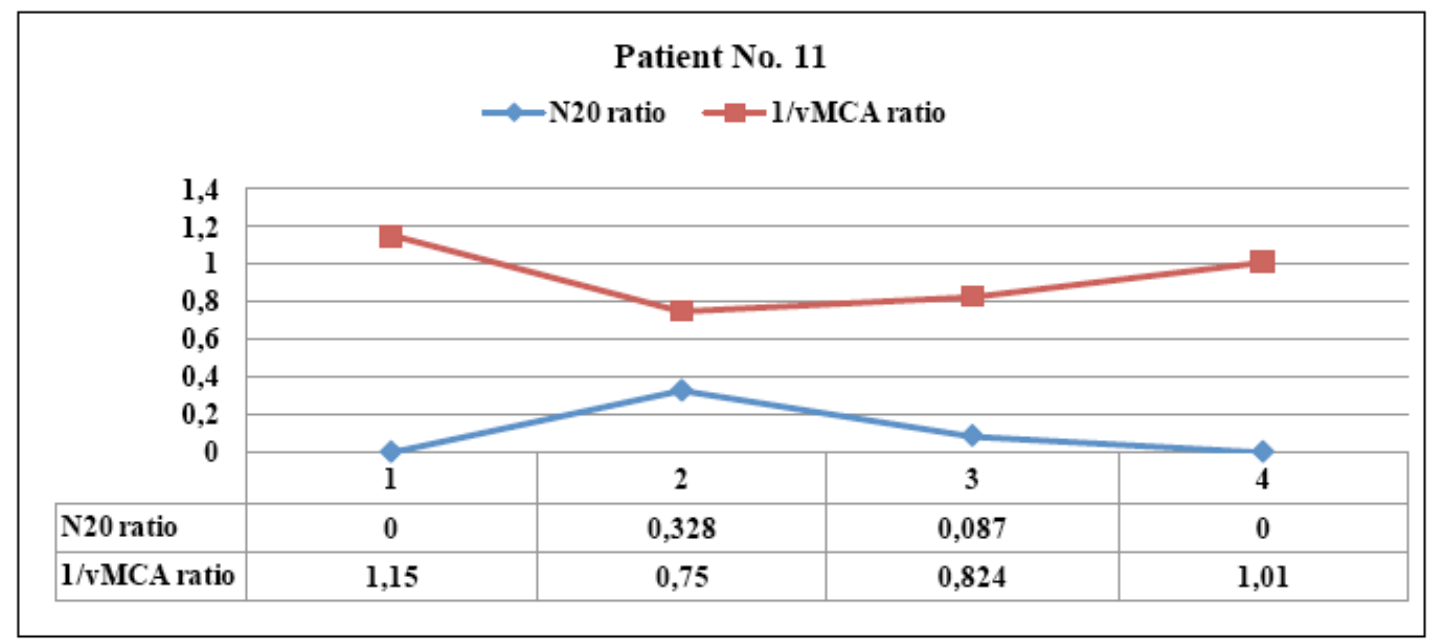

Fig. (9). This patient showed left parietal hemorrhagic damage evident at CT scan immediately after SAH: the courses of N20 and 1/vMCA ratios were divergent and did not correlate $(\mathrm{p}=0.89)$, since $\mathrm{N} 20$ had pathologic amplitude $<1.2 \mu \mathrm{V}$ at point 1 and 2 and disappeared in subsequent examinations, whereas 1/vMCA was always almost symmetric on hemispheres. The patient never had vasospasm and clinical stable hemiparesis was evident.

normal. On the other hand, if both low ratio values persist, as shown in patient 2, who reached interhemispheric values $<0.4$ for three consecutive days, structural damage develops. When evident CT/MRI structural brain damage occurs subsequently (patient No.2), or immediately after SAH (patients No. 4, 8, 11), there is a lack of correlation, as pathologic amplitude $(<1,2 \mu \mathrm{V})$ or absence of $\mathrm{N} 20$, on the compromised side, reflects the tissue damage and determined stable interhemispheric ratio $<0.65$. Conversely, in this situation $1 / \mathrm{vMCA}$ reverted to a ratio $>0.65$ with respect to the contralateral. This lack of correlation is probably due to the loss of neurovascular coupling and suggests a luxury perfusion in ischemic areas which no longer vasoregulate. The three patterns described above are shown in the scatter diagram (Fig. 12). A previous study investigated correlation between SSEP Central Conduction Time (CCT) and vasospasm and found a statistically significant $(\mathrm{p}<0.01)$ increase in actual CCT $(6.7$ $\mathrm{msec}$ ) in only the severe grade of vasospasm (blood flow velocity $>200 \mathrm{~cm} / \mathrm{s}$ ) in subarachnoid hemorrhage [24]. By contrast, in our approach, variations of $1 / \mathrm{vMCA}$ and $\mathrm{N} 20$ cortical amplitude showed correlation before damage developed and independently from vasospasm. The high correspondence between variation in vascular resistances due to vessel section and N20 amplitude can be explained by the close correlation between blood flow and electric activity in the cortical areas. In fact, the cortex, whose activity is expressed by the $\mathrm{N} 20$ amplitude, has greater ischemic sensitivity than the thalamus and medial lemniscus, which are involved in CCT [25]. In an experimental study of graded cerebral ischemia on an animal model, Burnett and colleagues demonstrated that the reduction in SSEP amplitude corresponds to a progressive regional $\mathrm{CBF}$ ( $\mathrm{rCBF}$ ) decrease and even a small reduction of as little as $10 \%$ in $\mathrm{rCBF}$ produces analogue modification in SSEP [26]. Similarly, in healthy subjects, linear coupling and linear covariation between N20-P22 amplitude and intensity of blood oxygen level-dependent functional magnetic resonance imaging (BOLD- fMRI) has been demonstrated [27, 28]. Correspondence between variations of 1/vMCA and N20 cortical amplitude in our data emerges whether vasospasm is detected or not, as it was detected in $23 \%$ of examinations of group 1 and in $57 \%$ of group 2 . Recent studies reviewed by Pluta and colleagues suggest that vasospasm of larger arteries is only to be an epiphenomenon of early brain injury after SAH with consequent microvascular vasospasm, spreading depression and cortical dysfunction [8] and the difficulty in defining the correlation between vasospasm and ischemia has been highlighted: perfusion abnormality without macrovascular vasospasm in the watershed areas or in the vicinity of sulcal clots has been demonstrated [29]. Dankbaar and colleagues reported that vasospasm decreases cerebral perfusion, but corresponds with the least perfused region in only two thirds of patients; furthermore, almost half of patients with severe vasospasm do not have delayed cerebral ischemia (DCI), and, although severe vasospasm can decrease perfusion, it may not result in DCI [30]. Carrera and colleagues found that $40 \%$ of patients who developed DCI did not reach blood flow velocity $(\mathrm{BFV})>120 \mathrm{~cm} / \mathrm{sec}$ and $16 \%$ of patients never registered $\mathrm{BFV}>120$ [31]. Some limitations of our study have to be considered. First, we considered a small number of patients and examinations (SSEP,TCCD and neuroimaging) were timed on the clinical basis evaluation, not following a specific protocol. Second, the semi quantitative comparison of data from both hemispheres can be missed if data change to the same pathologic extent bilaterally. On the basis of our approach, symmetric bilateral pathological variation of vascular resistances of MCA would correspond to analogue symmetric variation of N20 amplitude: in this case we could not evaluate the ischemic risk by only comparing the two ratios. We can hypothesize that a very low bilateral N20 amplitude, close to the pathological threshold of $1,2 \mu \mathrm{V}$, could be detected; however we found no such condition of symmetric bilateral pathological changes in our case series. Third, we evaluated modifications of MCA brain supplied area by CT or MRI, who detect changes with a different sensitivity; moreover, recently Dankbaar and colleagues demonstrated that CT perfusion (CTP) is more sensitive in detecting ischemic penumbra than CT [32], so its use could help to better define the three interhemispheric patterns of neurovascular coupling. 


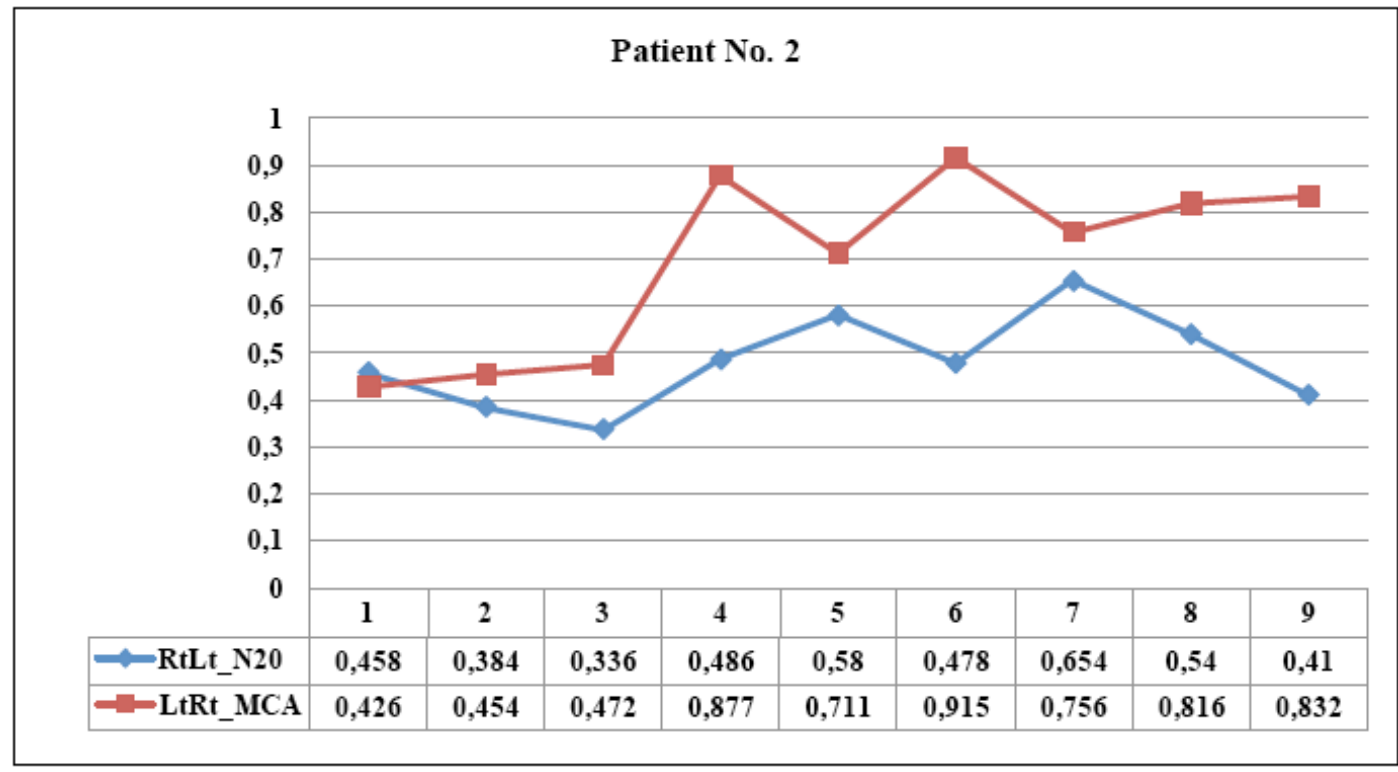

$\mathbf{a}$

\section{Patient No. 2}

Point 2

Exam n. 3 in tab. 3

Rt/Lt N20 ratio $=0.384$
Point 4

Exam n. 5 in tab. 3

Rt/Lt N20 ratio $=0.485$
Point 8

Exam n. 9 in tab. 3
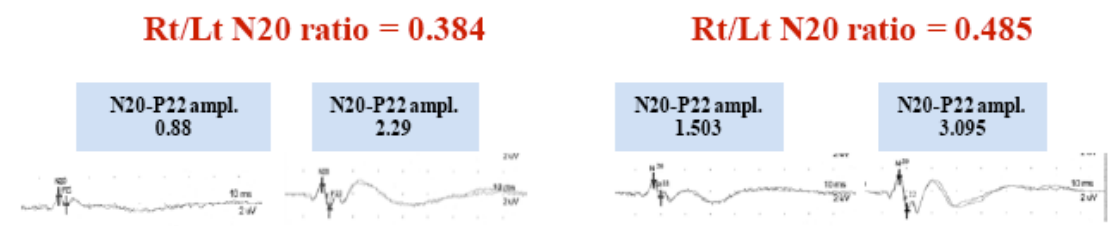

$\mathrm{Rt} / \mathrm{Lt} \mathrm{N} 20$ ratio $=0.541$
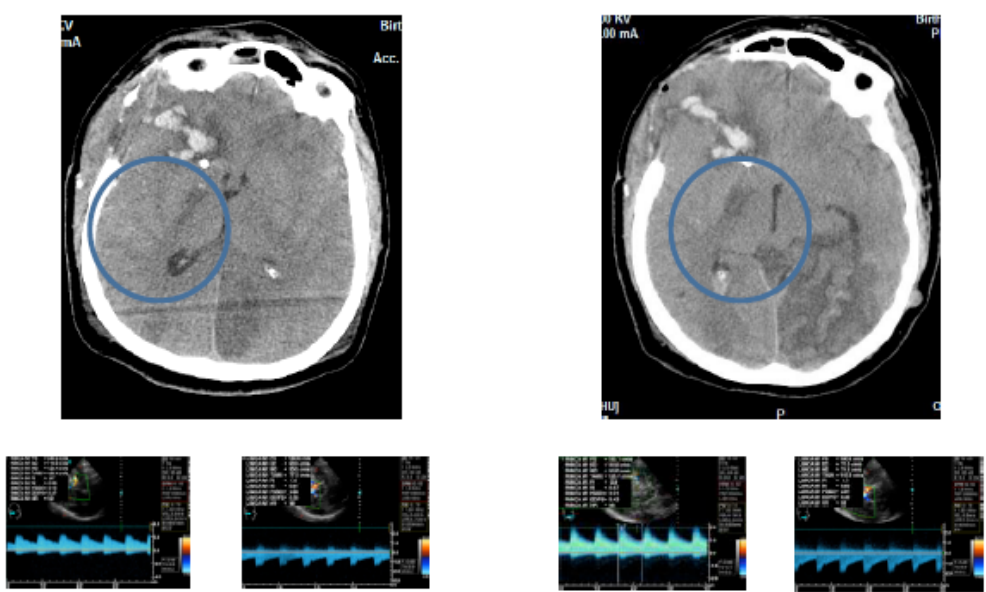

Rt 1/vMCA :

1/169.4

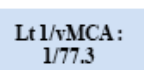

Rt 1/vMCA :

1/125.7

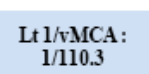

Rt $/$ Lt 1/vMCA ratio $=0,877$

b
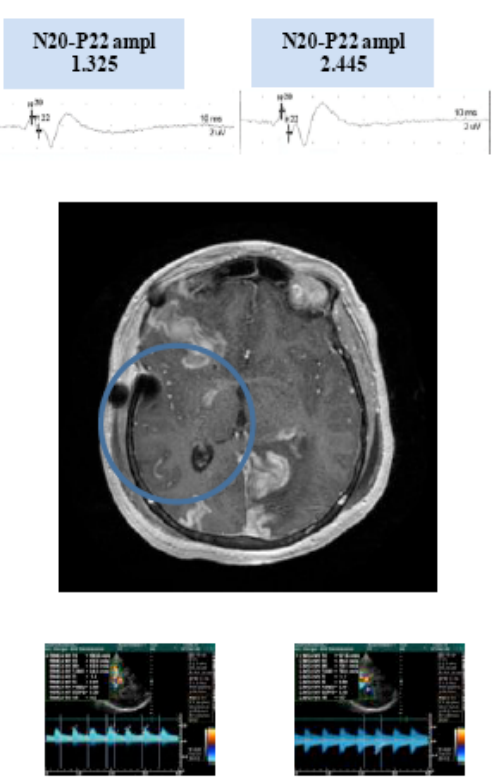

Lt 1/vMCA :

$1 / 94.2$

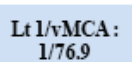

Rt $/$ Lt $1 /$ vMCA ratio $=0.816$

Rt $/$ Lt $1 /$ vMCA ratio $=0.456$

Fig. (10). Patient No. 2 showed parallel courses of very low N20 and 1/v MCA ratio $(<0.65)$ detected in the first three examinations $(\mathbf{a}$, points 1 to 3), corresponding with CT image of hypodesity of the right capsula (b, point 2); in the subsequent examinations, the divergent courses of $\mathrm{N} 20(<0.65)$ and 1/vMCA $(>0.65)$ and non correlated ratios $(\mathrm{p}=0.2 \mathrm{r}=0.46$, C.I. for $\mathrm{r}:-0.28$ to 0.86$)$ corresponded with CT and MRI images of structural infarct in the same area (b, point 4 and 8). Right N20 amplitude was $<1.2 \mu \mathrm{V}$ at points 1 to 3 and 6 to 7 (see examinations $2,3,4,7,8$ in Table 3) and $=<1.5 \mu \mathrm{V}$ at points 4,5, 8and 9 (see examinations 5,6,9,10 in Table 3); right MCA vasospasm (BFV $>120<180$ $\mathrm{cm} / \mathrm{sec}$ ) was detected at points 1 to 6 . Clinical hemiparesis was always evident. (N20 amplitude is expressed in $\mu \mathrm{V}$ and MCA blood flow velocity in $\mathrm{cm} / \mathrm{sec})$. 


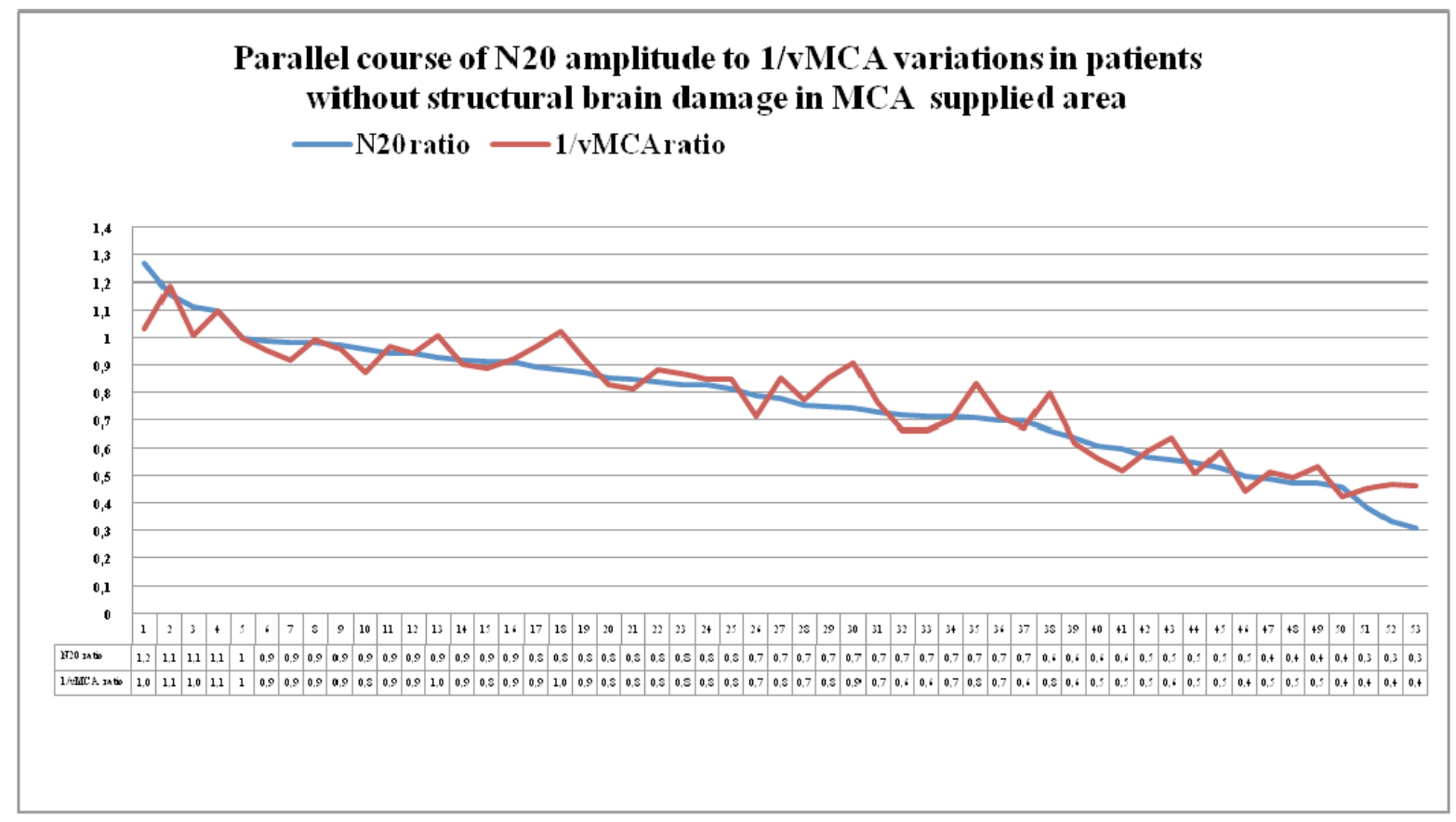

Fig. (11). Couples of N20 and 1/vMCA ratio values detected in patients of group 1 and group 2, who had no CT/MRI image of structural ischemic or haemorrhagic injury in MCA supplied area, displayed in descending order. Modifications of interhemispheric ratio of $1 / \mathrm{vMCA}$ correspond with analogue variations in interhemispheric ratio of $\mathrm{N} 20$ amplitude in a wide range of values, from 0.9 to $0.4-0.3,(\mathrm{p}<0.01, \mathrm{r}=$ 0.93, $\mathrm{Cl}$ for $\mathrm{r}=0.89$ to 0.96 unless structural ischemic or haemorrhagic damage develops.

\section{Patterns of $1 / \mathrm{vMCA}$ and N20 interhemispheric ratio related to CT/MRI scan images of corresponding MCA area}

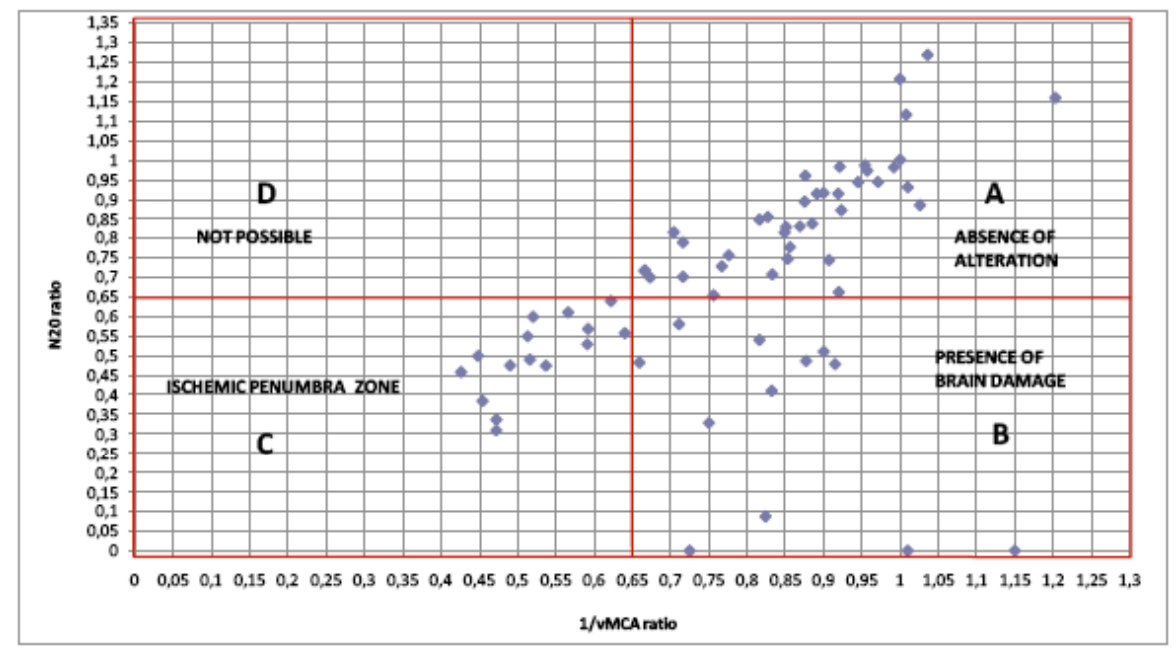

Fig. (12). The scatter diagram shows the distribution of all 66 couples of 1/vMCA and N20 ratio values gathered in Table 3. Each point identifies a couple of 1/vMCA (X axis) and N20 (Y axis) interhemispheric ratio values. In section A, both 1/vMCA and N20 interhemispheric ratio values $>0.65$ correspond to CT/MRI normal images of MCA supplied area: this is the area of absence of damage, where N20 amplitude and 1//vMCA values show interhemispheric symmetry. In section B, 1/vMCA ratios > 0.65 coupled with N20 ratios < 0.65 correspond with CT/MRI images of unilateral structural brain damage in the MCA supplied area: this is the brain injury area, where pathological amplitude or absence of N20 reflects the tissue damage, whereas 1/vMCA values reverting to those of the uninjured contralateral area suggests a luxury perfusion state. In section C, both 1/vMCA and N20 interhemispheric ratio values $<0.65$ correspond with CT/MRI images of unilateral transient and reversible alterations in MCA supplied area; this condition is capable of reverting to normal (section $\mathbf{A}$ ) or evolving to stable damage (section B): this section could be named " ischemic penumbra zone". In section D no ratio allocation is found: this area would correspond with a condition of unilateral hemispheric alteration of vascular resistances due to reduction of MCA vessel section and consequent low $1 / \mathrm{v}$ MCA ratio, in contrast to symmetrical bilateral N20 amplitude and high interhemispheric ratio value. It is reasonable to hypothesize an impossible coexistence of altered regional brain perfusion with normal cortical activity. 


\section{CONCLUSION}

MCA vascular changes detected by TCCD in SAH show significant statistic linear correlation with cortical activity measured by the early cortical component (N20) of the somatosensory evoked potential from the median nerve. Three different patterns of neurovascular coupling were identified matching the interhemispheric ratios of N20 and reciprocal of MCA mean flow velocity detected within each patient, to CT scan or MRI images of MCA areas. 1) 1/vMCA and N20 ratios $>0.65$ correspond with absence of neuroimaging alterations in MCA areas and lateralizing symptoms: this pattern indicates an ongoing equilibrium of neurophysiological and vascular condition. 2): both $1 / \mathrm{vMCA}$ and $\mathrm{N} 20$ values lower than 0.65 in respect of the contralaterals correspond with TC/MRI scan images of transient alterations in the MCA area and clinical evidence of lateralizing symptoms or consciousness modification: this pattern identifies a situation of reversible ischemic penumbra and can evolve either to the re-establishment of a normal and symmetric condition, expressed in pattern 1, where N20 and 1/vMCA absolute values show little or no interhemispheric difference; or to the next pattern. 3) 1/vMCA value $>0.65$ and N20 amplitude $<$ 0.65 in respect of the contralaterals correspond with TC/MRI scan images of hemispheric structural damage in the MCA area: this is a condition of evident structural ischemic or hemorrhagic damage in which there is a luxury perfusion in the injured brain area. Although more studies are required to confirm our hypothesis, we would like to emphasize the importance of simultaneous evaluation of somatosensory evoked potential and the TCCD to monitor the neurovascular coupling and to detect the ischemic penumbra due to MCA vascular changes in $\mathrm{SAH}$, before the ischemic damage develops.

\section{CONSENT}

Written consent was obtained from the patients for publication of these case series and any accompanying images. A copy of the written consent is available for review from the Editor-in-Chief of this journal.

\section{AUTHORS' CONTRIBUTIONS}

PDP conceived the work, collected and analysed the data as well as collaborated in writing the article. PZ analyzed the data and collaborated in writing the article. MI analyzed the data and collaborated in writing the article. EB analyzed the data. EF analyzed the data and elaborated the statistical calculations. All authors have read and approved the final manuscript.

\section{ACKNOWLEDGEMENTS}

The authors wish to thank the teaching staff of Florence University Neurophisiology Department, in particular Pinto F. $(\mathrm{PhD})$.The authors also extend their thanks the Rovigo Hospital ward director, Palù M. (MD), whose collaboration enabled the study to be carried out.

The manuscript has been proof read and corrected by Sheona Balish M.A. (ord), TOEFL qualified, a native English speaker.

\section{ABBREVIATIONS}

MCA $=$ middle cerebral artery

$\begin{array}{ll}\text { TCCD } & =\text { Transcranial color Doppler } \\ \text { SSEP } & =\text { somatosensory evoked potentials } \\ \text { SAH } & =\text { inverse of Middle Cerebral Artery mean } \\ \text { 1/vMCA } & =\text { blood flow velocity } \\ \text { DSA } & =\text { cerebral perfusion pressure } \\ \text { CPP } & =\text { cerebral blood flow } \\ \text { CBF } & =\text { blood flow velocity } \\ \text { rCBF } & =\text { Computed Tomographic angiography } \\ \text { BFV } & =\text { Computed Axial Tomography } \\ \text { CTA } & =\text { Magnetic Resonance Imaging } \\ \text { CT } & =\text { External Ventricular Drainage } \\ \text { MRI } & =\text { blood oxygen level-dependent functional } \\ \text { EVD } & =\text { magnetic resonance imaging } \\ \text { BOLD- fMRI } & \text { Statistical Analysis System } \\ \text { SAS } & \text { SPSS }\end{array}$

\section{REFERENCES}

[1] Krejza J, Mariak Z, Walacki J, Szydlik, Lewko J, Ustymowicz A. Transcranial Color Doppler Sonography of Basal Cerebral Arteries in 182 Healthy Subjects: Age and Sex variability and Normal References Values for Blood Flow Parameters. AJR Am J Roentgenol 1999; 172: 213-8.

[2] Bartels E, Fuchs HH, Flügel KA. Color Doppler Imaging of basal cerebral arteries: normal reference values and clinical applications. Angiology 1995; 46: 877-84.

[3] van de Wassenberg WJ, van der Hoeven JH, Leenders KL, Maurits NM. Quantifying interhemispheric symmetry of somatosensory evoked potentials with the intraclass correlation coefficient. J Clin Neurophysiol 2008; 25: 139-46.

[4] Connemann BJ, Koehler J, Presser S, Hopf HC. Latency and amplitude variability in serial median nerve SEP recordings. Clin Neurophysiol 1999; 110: 1664-8.

[5] Soehle M, Czosnyka M, Pickard JD, Kirkpatrick PJ. Continuous assessment of cerebral autoregulation in subarachnoid hemorrhage. Anesth Analg 2004; 98: 1133-9.

[6] Lang EW, Diehl RR, Mehdorn HM. Cerebral autoreguation testing after aneurismal subarchnoid haemorrhage: the phase relationship between arterial blood pressure and cerebral blood flow velocity. Crit Care Med 2001; 29: 158-63.

[7] Macdonald RL, Pluta RM, Zhang JH. Cerebral vasospasm after subarachnoid hemorrhage: the emerging revolution. Nat Clin pract Neurol 2007; 3: 256-63.

[8] Pluta RM, Hansen-Schwartz J, Dreier J, et al. Cerebral vasospasm following subarachnoid hemorrhage: time for a new world of thought. Neurol Res 2009; 31: 151-8.

[9] Branston NM, Symon L, Crockard HA, Pastzor E. Relationship between the cortical evoked potential and local cortical blood flow following acute middle cerebral artery occlusion in the baboon. Exp Neurol 1974; 45: 195-208.

[10] Kang DZ, Wu ZY, Lan Q, et al. Combined monitoring of evoked potentials during microsurgery for lesions adjacent to the brainstem and intracranial aneurysms. Chin Med J 2007; 120: 1567-73. 
[11] Penchet G, Arné P, Cuny E, Monteil P, Loiseau H, Castel JP. Use of intraoperative monitoring of somatosensory evoked potentials to prevent ischemic stroke after surgical exclusion of middle cerebral artery aneurysms. Acta Neurochir 2007; 149: 357-64.

[12] Florence G, Guerit JM, Gueguen B. Electroencephalography (EEG) and somatosensory evoked potentials (SEP) to prevent cerebral ischemia in the operating room. Neurophysiologie clinique 2004; 34: 17-32.

[13] Diringer MN. Management of aneurysmal subarachnoid hemorrhage. Crit Care Med 2009; 37: 432-40.

[14] Schubert GA, Thome C. Cerebral blood flow changes in acute subarachnoid haemorrhage. Front Biosci. 2008; 13: 1594-603.

[15] Losiniecki A. Zuccarello M. Subarachnoid haemorrhage: effect on cerebral blood flow and cerebral metabolism. Front Biosci 2008; 13: 1845-56.

[16] Keyrouz SG, Diringer MN. Clinical review: Prevention and therapy of vasospasm in subarachnoid hemorrhage. Crit Care 2007; 11: 220.

[17] Sehba FA, Bederson JB. Mechanisms of acute brain injury after subarachnoid haemorrhage. Neurol Res 2006; 28: 381-98.

[18] Munch E, Vajkoczy P: Current advances in the diagnosis of vasospasm. Neurol Res 2006; 28: 703-12.

[19] Wartenberg KE, Schmidt JM, Mayer SA. Multimodality Monitoring in Neurocritical Care. Crit Care Clin 2007; 23: 507-38.

[20] Geeraertsa T, Leblanca PE, Dufoura G, Tazarourteb K, Duranteaua J, Viguéa B. Monitorage intracérébral d'un patient ayant un vasospasme. Ann Fr Anesth Reanim 2007; 26: 973-9.

[21] Amantini A, Grippo A, Fossi S, et al. Prediction of 'awakening' and outcome in prolonged acute coma from severe traumatic brain injury: evidence for validity of short latency SEPs. Clinical Neurophysiology 2005; 116: 229-35.

[22] Aaslid R, Huber P, Nornes H. Evaluation of cerebrovascular spasm with transcranial Doppler ultrasound. J Neurosurg 1984; 60: 37-41.

[23] Lindegaard KF. The role of transcranial Doppler in the management of patients with subarachnoid haemorrhage: a review. Acta Neurochir Suppl 1999; 72: 59-71.
[24] Szabó S, Mikó L, Novák L, Rózsa L, Székely G Jr. Correlation between central somatosensory conduction time, blood flow velocity, and delayed cerebral ischemia after aneurysmal subarachnoid hemorrhage. Neurosurg Rev 1997; 20: 188-95.

[25] Branston NM, Ladds A, Symon L, Wang AD. Comparison of the effects of ischaemia on early components of the somatosensory evoked potential in brainstem, thalamus, and cerebral cortex. J Cereb Blood Flow Metab 1984; 4: 68-81.

[26] Burnett MG, Detreb JA, Greenbergb JH. Activation-flow coupling during graded cerebral ischemia. Brain Res 2005, 1047: 112-8.

[27] Arthurs O J, Williams EJ, Carpenter TA, Pikard JD, Boniface SJ. Linear coupling between functional magnetic resonance imaging and evoked potential amplitude in human somatosensory cortex. Neuroscience 2000; 101: 803-6.

[28] Arthurs OJ, Donovan T, Spiegelhalter DJ, Pickard JD, Boniface SJ. Intracortically Distributed Neurovascular Coupling Relationships within and between Human Somatosensory Cortices. Cerebral Cortex March 2007; 17: 661-8.

[29] Aralasmak A, Akyuz M, Ozkaynak C, Sindel T, Tuncer R. CT angiography and perfusion imaging in patients with subarachnoid hemorrhage: correlation of vasospasm to perfusion abnormality. Neuroradiology 2009; 51: 85-93.

[30] Dankbaar JW, Rijsdijk M, van der Schaaf IC, Velthuis BK, Wermer M JH, Rinkel GJE. Relationship between vasospasm, cerebral perfusion, and delayed cerebral ischemia after aneurismal subarachnoid hemorrhage. Neuroradiol 2009; 51: 813-9.

[31] Carrera E, Schmidt JM, Oddo M, et al. Transcranial Doppler for predicting delayed cerebral ischemia after subarachnoid hemorrhage. Neurosurgery 2009; 65: 316-23.

[32] Dankbaar JW, de Rooi JNK, Velthuis BK, Frijns CJ, Rinkel GJE, van der Schaaf IC. Diagnosing delayed cerebral ischemia with different CT modalities in patients with subarachnoid hemorrhage with clinical deterioration. Stroke 2009; 40: 3493-8.

(c) Di Pasquale et al.; Licensee Bentham Open.

This is an open access article licensed under the terms of the Creative Commons Attribution Non-Commercial License (http://creativecommons.org/licenses/by-nc/3.0/) which permits unrestricted, non-commercial use, distribution and reproduction in any medium, provided the work is properly cited. 